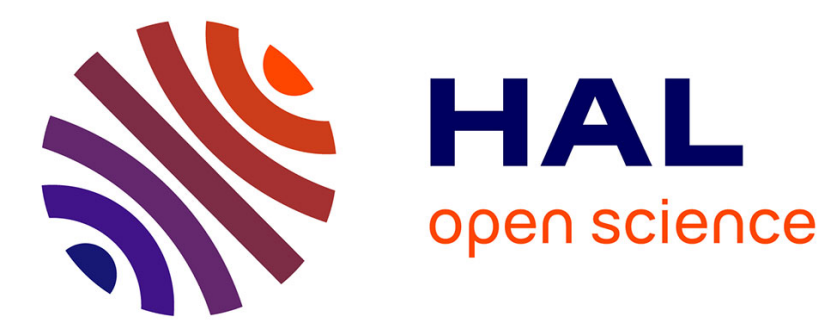

\title{
Criteria of Divergence Almost Everywhere in Ergodic Theory \\ Michel Weber
}

\section{To cite this version:}

Michel Weber. Criteria of Divergence Almost Everywhere in Ergodic Theory. Journal of Mathematical Sciences, 2016, 219 (5), pp.651-682. 10.1007/s10958-016-3137-y . hal-02419521

\section{HAL Id: hal-02419521 \\ https://hal.science/hal-02419521}

Submitted on 19 Dec 2019

HAL is a multi-disciplinary open access archive for the deposit and dissemination of scientific research documents, whether they are published or not. The documents may come from teaching and research institutions in France or abroad, or from public or private research centers.
L'archive ouverte pluridisciplinaire HAL, est destinée au dépôt et à la diffusion de documents scientifiques de niveau recherche, publiés ou non, émanant des établissements d'enseignement et de recherche français ou étrangers, des laboratoires publics ou privés. 
Journal of Mathematical Sciences, Vol. 00, No. 0, Yyyy, 0000

\title{
CRITERIA OF DIVERGENCE ALMOST EVERYWHERE IN ERGODIC THEORY
}

\begin{abstract}
M. J. G. Weber*
UDC 519.2

In this expository paper, we survey nowadays classical tools or criteria used in problems of convergence everywhere to build counterexamples: the Stein continuity principle, Bourgain's entropy criteria, and Kakutani-Rokhlin lemma, the most classical device for these questions in ergodic theory. First, we state a $L^{1}$-version of the continuity principle and give an example of its usefulness by applying it to a famous problem on divergence almost everywhere of Fourier series. Next we particularly focus on entropy criteria in $L^{p}, 2 \leq p \leq \infty$, and provide detailed proofs. We also study the link between the associated maximal operators and the canonical Gaussian process on $L^{2}$. We further study the corresponding criterion in $L^{p}, 1<p<2$, using properties of $p$ stable processes. Finally, we consider Kakutani-Rokhlin's lemma, one of the most frequently used tools in ergodic theory, by stating and proving a criterion for a.e. divergence of weighted ergodic averages. Bibliography: 38 titles.
\end{abstract}

\section{INTRODUCTION}

This is an expository paper on criteria of divergence almost everywhere in ergodic theory, and mainly Bourgain's entropy criteria in $L^{p}, 2 \leq p \leq \infty$. The paper is written in a selfcontained and informative way: tools needed are presented, with (expected to be) helpful and sometimes historical comments, auxiliary results are included, as well as detailed and careful proofs of main theorems. The preparation of this paper is thus made in order to be also an efficient tool for investigating these questions. This is, in fact, our main objective. We do not study nor present applications. We refer for these to Bourgain [1-3]. We also refer to Rosenblatt and Wierdl's monograph [26], to our monograph [33] devoted to the study of these criteria, and to Chaps. 5 and 6 of our book [32], where applications of the Stein continuity principle are also studied. We further refer to Lacey [18], Lesigne [20], and Berkes and Weber [6] notably for other applications. In writing the present paper, we referred to Chap. 6 of [32]. We were able to improve and simplify some proofs and also complete it by new results. The entropy criterion in $L^{p}, 1<p<2$, obtained in Weber [38] is stated and proved under a less restrictive commutation assumption, and we included the necessary material from the theory of $\alpha$-stable processes (here $\alpha=p$ ) for the proof. The metric entropy method (first introduced by Strassen in the theory of Gaussian processes, see [8]) is briefly and concisely presented for the need of the study.

The paper is organized as follows. In Sec. 2, we start with what is certainly, by the probabilistic argument used in its proof, the basis of everything: the Stein continuity principle. A less known aspect of this principle is that it is also a tool for producing counterexamples to almost everywhere questions. That point is developed in this section. Next, Sec. 3 is the central part of the paper and concerns Bourgain's entropy criteria and extensions of them. In Sec. 4 , we present auxiliary results concerning $L^{p}$-isometries, stable random variables and processes, variants of Banach principle, a metric comparison lemma, and basic Gaussian tools. Section 5 is completely devoted to proofs of the results stated in Sec. 3. We conclude the paper with Kakutani-Rokhlin lemma, one of the most classical devices in ergodic theory. There are many applications of this result, also called Kakutani-Rokhlin towers' lemma. We refer to

*IRMA, Université Louis-Pasteur et C.N.R.S., Strasbourg, France, e-mail: michel . weber@math.unistra.fr.

Translated from Zapiski Nauchnykh Seminarov POMI, Vol. 441, 2015, pp. 73-118. Original article submitted November 12, 2015. 
Rosenblatt and Wierdl's monograph [26]. We illustrate it by stating and proving a criterion for a.e. divergence of weighted ergodic averages, based on Deniel's construction [7].

\section{The Continuity Principle}

Let $(X, \mathcal{A}, \mu)$ be a probability space with a $\mu$-complete $\sigma$-field $\mathcal{A}$. Throughout the paper, $S$ denotes, unless explicitly mentioned, a sequence of continuous operators $S_{n}: L^{2}(\mu) \rightarrow L^{2}(\mu)$, $n \geq 1$. Recall some basic facts. Let $1 \leq p \leq \infty$. By the Banach principle, the set

$$
\mathcal{F}(S)=\left\{f \in L^{p}(\mu):\left\{S_{n} f, n \geq 1\right\} \text { converges } \mu \text {-almost everywhere }\right\}
$$

is closed in $L^{p}(\mu)$ if and only if:

There exists a nonincreasing function $C: \mathbb{R}^{+} \rightarrow \mathbb{R}^{+}$with $\lim _{\alpha \rightarrow \infty} C(\alpha)=0$ and such that for any $\alpha \geq 0$ and any $f \in L^{p}(\mu)$,

$$
\mu\left\{S^{*} f>\alpha\|f\|_{p}\right\} \leq C(\alpha), \quad \text { where } \quad S^{*} f=\sup _{n \geq 1}\left|S_{n} f\right| .
$$

When the sequence $S$ commutes with a sequence $\left\{\tau_{j}, j \geq 1\right\}$ of measurable transformations of $X$ preserving $\mu$ and mixing in the following sense:

$$
\begin{aligned}
& \text { for any } A, B \in \mathcal{A} \text { and any } \alpha>1, \text { there exists } j \geq 1 \\
& \text { such that } \mu\left(A \cap \tau_{j}^{-1} B\right) \leq \alpha \mu(A) \mu(B)
\end{aligned}
$$

and $1 \leq p \leq 2$, then, by the continuity principle, $C(\alpha)=\mathcal{O}\left(\alpha^{-p}\right)$.

This is fulfilled if $S$ commutes with an ergodic endomorphism of $(X, \mathcal{A}, \mu)$. So that the study of the convergence almost everywhere of the sequence $S$ amounts, under appropriate commutation assumptions, to establish a maximal inequality and to exhibit a dense subset of $L^{p}(\mu)$ for which the convergence almost everywhere already holds.

Before stating the Continuity Principle, recall that the topology of convergence in measure on $L^{0}(\mu)\left(g_{n} \stackrel{\mu}{\rightarrow} g\right.$ if $\mu\left\{\left|g_{n}-g\right|>\varepsilon\right\} \rightarrow 0$ for any $\left.\varepsilon>0\right)$ is metrizable and, endowed with the metric $d(f, g)=\int_{X} \frac{|f-g|}{1+|f-g|} d \mu,\left(L^{0}(\mu), d\right)$ is a complete metric space. A mapping $V$ from a Banach space $B$ to $L^{0}(\mu)$ is said to be continuous in measure, or $d$-continuous, if $d\left(S f_{n}, S f\right) \rightarrow 0$ for any sequence $\left(f, f_{n}, n \geq 1\right) \subset B$ whenever $\left\|f_{n}-f\right\| \rightarrow 0$.

Theorem 2.1. Assume that $\left\{S_{n}, n \geq 1\right\}$ is a sequence of operators, $S_{n}: L^{p}(\mu) \rightarrow L^{0}(\mu)$, $1 \leq p \leq 2$, that are continuous in measure and satisfy the commutation assumption $(H)$. Then the following properties are equivalent:

(i) for any $f \in L^{p}(\mu), \quad \mu\left\{x: S^{*} f(x)<\infty\right\}=1$;

(ii) there exists a constant $0<C<\infty$ such that for any $f \in L^{p}(\mu)$,

$$
\sup _{\lambda \geq 0} \lambda^{p} \mu\left\{x: S^{*} f(x)>\lambda\right\} \leq C \int_{X}|f|^{p} d \mu .
$$

Remark 2.2. If $p>2$, the same conclusion holds for positive operators $\left(S_{n} f \geq 0\right.$ if $f \geq 0$ ). This was proved later by Sawyer in [27].

The proof combines, quite subtely and remarkably, analysis and probability. The commutation property of the operators $S_{n}$ is crucial and makes the proof possible. Earlier, Kolmogorov used already in [13] the fact that the operators

$$
H_{n} f(x)=\int_{|t|>1 / n} f(x-t) \frac{d t}{t}, \quad f \in L_{\mathrm{loc}}^{1}(\mathbb{R}),
$$


all commute with translations to prove a similar inequality: if $H^{*} f(x)=\sup \left\{\left|H_{n} f(x)\right|, n \geq 1\right\}$, then

$$
\sup _{\lambda \geq 0} \lambda m\left\{x: H^{*} f(x)>\lambda\right\} \leq C \int_{\mathbb{R}}|f(x)| d x,
$$

where $m$ denotes the Lebesgue measure on $\mathbb{R}$. The setting considered in [29] is group theoretic: $\Omega$ is a commutative compact group, $\mu$ is the Haar measure, and $S_{n}$ are commuting with translations. Sawyer [27] showed that this setting is not necessary and that a general principle can be derived under the above assumptions. We refer to the nice monograph of Garsia [10].

The Continuity Principle is not only a tool for studying integrability of maximal operators $S^{*} f$, but also a device for producing counterexamples in problems of convergence almost everywhere. This was already observed and studied by Stein [29], but also by Burkholder [5] and Sawyer [27]. In [29], Stein has established other forms of this principle with quite striking applications, proving notably negative convergence results. One of these applications concerns a deep result of Kolmogorov [14,15] showing the existence of an integrable function whose Fourier series diverges almost everywhere. The proof is known to be very difficult. Using a suitable form of his principle for the space $L^{1}(\mu)$, Stein could refine and also provide a simpler proof of Kolmogorov's result. Convergence criteria for this space are not frequent, and reveal crucial in many deep questions. We recall it now.

We assume here that $X$ is a commutative compact group and denote by "+" the group operation. Let $\mu$ be the unique invariant measure, the Haar measure on $X$. Let $\mathcal{C}(X)$ be the space of continuous functions on $X$ with the supremum norm and let $\mathcal{B}(X)$ be the space of finite Borel measures on $X$ with the usual norm. Let $\left\{S_{n}, n \geq 1\right\}$ be a sequence of operators. We assume that:

(a) Each $S_{n}$ is a bounded operator from $L^{1}(\mu)$ to $\mathcal{C}(X)$.

(b) Each $S_{n}$ commutes with translations.

By Riesz's representation of bounded linear functionals on $L^{1}(\mu)$, conditions (a) and (b) are equivalent to

(c) $S_{n} f(x)=\int_{X} K_{n}(x-y) f(y) \mu(d y)$, where $K \in L^{\infty}(X)$.

Such an operator has a natural extension to a bounded operator from $\mathcal{B}(X)$ to $L^{\infty}(\mu)$, which we again denote by $S_{n}$. Notice that this extension still commutes with translations. Similarly, we also write $S^{*} \nu=\sup _{n \in \mathbb{N}}\left|S_{n} \nu\right|$.

Theorem 2.3. Under assumptions (a) and (b), the following assertions are equivalent:

$$
\text { for any } f \in L^{1}(\mu), \quad \mu\left\{x: S^{*} f(x)<\infty\right\}=1 \text {; }
$$

there exists a constant $0<C<\infty$ such that for any $\nu \in \mathcal{B}(X)$,

$$
\sup _{\lambda \geq 0} \lambda \mu\left\{x: S^{*} \nu(x)>\lambda \int_{X}|d \nu|\right\} \leq C .
$$

To give an idea of its strength, let us show how to recover Kolmogorov's theorem. Introduce the necessary notation. We denote throughout this article by $\mathbb{T}$ the circle $\mathbb{R} / \mathbb{Z} \sim[0,1$.

Take $X=\mathbb{T}$ and let $\mu$ be the normalized Lebesgue measure on $\mathbb{T}$. Let $S_{n}(f)$ denote here the partial sum of order $n$ of the Fourier series of $f$, and, more generally, let $S_{n}(\nu)$ be the partial sum of order $n$ of the Fourier-Stieltjes expansion of a Borel measure $\nu$. Recall that for any integrable $f$,

$$
S_{n} f(x)-S_{m} f(x)=\mathcal{O}(\log (1+|m-n|)), \quad m, n \rightarrow \infty,
$$


almost everywhere. Stein proved the following refinement.

Theorem 2.4. Let $\varphi(n)>0$ be any function tending to zero as $n$ tends to infinity. Then there exists an integrable function $f(x)$ such that the more restrictive property,

$$
S_{n}(f)(x)-S_{m}(f)(x)=\mathcal{O}(\varphi(|m-n|) \log (1+|m-n|)),
$$

is false for almost every $x$.

This, of course, implies Kolmogorov's theorem. For the proof, consider for $n \neq m$ the family of operators

$$
\Delta_{(m, n)} f=\frac{S_{n}(f)-S_{m}(f)}{\varphi(|m-n|) \log (1+|m-n|)} .
$$

These operators satisfy conditions (a) and (b) of Theorem 2.3. A lemma is necessary.

Lemma 2.5. There exists an absolute constant $C$ such that for any integer $k$, there exists a measure $\nu$ on $\mathbb{T}$ with $\int_{\mathbb{T}}|d \nu|=1$ and such that

$$
\sup _{n, m:|n-m|=k}\left|S_{n}(\nu)-S_{m}(\nu)\right| \geq C \log k \quad \text { almost surely. }
$$

Proof. Let $x_{1}, \ldots, x_{N}$ be some points of $\mathbb{T}$ to be specified later, and set $\nu=\frac{1}{N} \sum_{i=1}^{N} \delta_{x_{i}}$, where $\delta_{x}$ denotes the Dirac measure at point $x$. Then $\int_{\mathbb{T}}|d \nu|=1$. Plainly,

$$
S_{n}(\nu)(x)-S_{m}(\nu)(x)=\frac{2}{N} \sum_{i=1}^{N} \frac{\cos \pi(n+m+1)\left(x-x_{i}\right) \sin \pi(n-m)\left(x-x_{i}\right)}{\sin \pi\left(x-x_{j}\right)} .
$$

Write $k=n-m$ and $\ell=n+m+1$. Assume that $k$ is odd. Then $\ell$ must be even, but this is the only restriction on $\ell$. We choose the $x_{i}$ to be linearly independent over $\mathbb{Q}$, and such that they are very close to $i / N$. It is easily seen then that for almost every $x$, the $x-x_{i}$ are linearly independent over $\mathbb{Q}$. Choosing $\ell$ large enough, depending on $x$, we have the equality

$$
\sup _{n, m:|n-m|=k}\left|S_{n}(\nu)(x)-S_{m}(\nu)(x)\right|=\frac{2}{N} \sum_{i=1}^{N} \frac{\left|\sin \pi k\left(x-x_{i}\right)\right|}{\left|\sin \pi\left(x-x_{j}\right)\right|} .
$$

The fact that $x_{i}$ are very close to $i / N$ and $N$ is large enough shows that the sum on the right is close to its integral counterpart, and so exceeds half of its value. Therefore,

$$
\sup _{n, m:|n-m|=k}\left|S_{n}(\nu)(x)-S_{m}(\nu)(x)\right| \geq \frac{1}{2} \int_{\mathbb{T}} \frac{|\sin \pi k(x-y)|}{|\sin \pi(x-y)|} d y \geq C \log k,
$$

as claimed.

Now we prove Theorem 2.4. Assume on the contrary that property (2.3) were true with positive probability, and this holds for any $f \in L^{1}(\mathbb{T})$. Let $\tau$ be an irrational rotation of $\mathbb{T}$, thereby, an ergodic measure preserving transformation. Note that if $A=\left\{\sup _{n \neq m}\left|\Delta_{(m, n)} f\right|<\right.$ $\infty\}$, then $\tau^{-1}(A) \subset A$. By Birkhoff's theorem, this suffices to imply that $\mu(A)=1$. So that the operators $\Delta_{(m, n)} f$ would satisfy condition (2.1). Consequently, the maximal operator

$$
\nu \mapsto \Delta^{*}(\nu):=\sup _{n \neq m}\left|\frac{S_{n}(\nu)(x)-S_{m}(\nu)(x)}{\varphi(|m-n|) \log (1+|m-n|)}\right|
$$

would satisfy (2.2). Therefore, this would imply the existence of a constant $C_{0}$ such that for any $\nu \in \mathcal{B}(M)$ with $\int_{\mathbb{T}}|d \nu|=1$ and any $t \geq 0, t \mu\left\{x: \Delta^{*} \nu(x)>t\right\} \leq C_{0}$. 
Let $k$ be a positive integer, which we choose sufficiently large to ensure that $\log k>\left(2 C_{0}\right) / C$, where $C$ is the same constant as in Lemma 2.5. Apply this for $t=(C \log k) / 2$; then

$$
\mu\left\{x: \Delta^{*} \nu(x)>\frac{C}{2} \log k\right\} \leq \frac{2 C_{0}}{C \log k}<1 .
$$

By Lemma 2.5, there exists a $\nu \in \mathcal{B}(M)$ with $\int_{\mathbb{T}}|d \nu|=1$ such that $\Delta^{*} \nu \geq C \log k$ almost surely. Hence, we get a contradiction, and condition (2.1) cannot hold. Therefore, there exists an integrable function such that property $(2.3)$ is false for almost every $x$.

For recent results related to Kolmogorov's theorem, see very nice Lacey's paper [17], Sec. 9.3. We refer to [29] (see also [32, Chap. 5]) for several other applications of that kind.

To $f \in L^{2}(\mu)$, associate the sequence in which we set $T_{j} f=f \circ \tau_{j}$,

$$
F_{J, f}=\frac{1}{\sqrt{J}} \sum_{1 \leq j \leq J} g_{j} T_{j} f, \quad J \geq 1,
$$

where $g_{1}, g_{2}, \ldots$ are i.i.d. standard Gaussian random variables defined on a common joint probability space $(\Omega, \mathcal{B}, \mathbf{P})$.

These random elements (with Rademacher weights instead of Gaussian) are key tools in Stein's proof. The same elements (sometimes with stable weights) are also playing a central role in Bourgain's entropy criteria and extensions obtained by the author. The notation used in (2.4) will be later formalized to include these cases, see (4.4). Lifshits and Weber studied in $[21,22]$ and [35] their oscillation properties and the tightness properties of their laws.

The Continuity Principle is established in an indirect way in [29]. A direct proof with Gaussian weights (as in the proofs of Bourgain's entropy criteria) was given in [32].

We close this section with an interesting and somehow intriguing observation. The key point of the proof is contained in the following inequality (see [32, p. 211-212]):

$$
\frac{n \mu\left\{S^{*}(f)>M(1+n)^{1 / p}\right\}-2}{n \mu\left\{S^{*}(f)>M(1+n)^{1 / p}\right\}} \leq 8 \mathbf{E} \mu\left\{S^{*}\left(F_{n, f}\right)>c M\right\},
$$

which holds for any $M>0$, any integer $n \geq 2$, and $c$ is a numerical constant. Now by simply permuting the order of integration, we get the inequality

$$
\frac{n \mu\left\{S^{*}(f)>M(1+n)^{1 / p}\right\}-2}{n \mu\left\{S^{*}(f)>M(1+n)^{1 / p}\right\}} \leq 8 \int_{X} \mathbf{P}\left\{S^{*}\left(F_{n, f}\right)>c M\right\} d \mu,
$$

where this time, $S^{*}(f)$ is controlled by its random counterpart of $S^{*}\left(F_{n, f}\right)$ for an appropriate choice of the integer $n$. Therefore, a good control of the random counterpart also provides a good control of the initial sequence.

Notation. We reserve the letter $g$ to denote throughout an $\mathcal{N}(0,1)$ distributed random variable. An index or a sub-index always denotes an infinite increasing sequence of positive integers.

\section{Metric Entropy Criteria}

Using the theory of Gaussian processes, Bourgain has established in [2] two very useful criteria linking regularity properties (boundedness, convergence almost everywhere) of a sequence $S$ with the metric entropy properties of the sets $C_{f}$ below.

The concept of entropy numbers (namely, covering numbers) associated with a metric space is old; it was invented by Kolmogorov as a device for classifying functional spaces. See Kolmogorov [13], Kolmogorov and Tikhomirov [16], and Lorentz [23]. In many situations, these 
numbers are computable (typical examples of sets are ellipsoids, see [9]); this explains their interest. Recall that any compact set in a separable Hilbert space is included in some ellipsoid, see Raimi [25], and for relations between their entropy numbers, see Helemskiu and Henkin [11].

Bourgain also showed, by means of imaginative constructions, how to apply these criteria to several analysis problems, among them Marstrand's disproof of Khintchin's Conjecture, a problem posed by Bellow, and a question raised by Erdös. This is a quite striking achievement, which adds a new chapter to Stein's Continuity Principle. We believe that Bourgain's approach goes beyond the setting explored in [1-3] and should deserve further investigations. The author has obtained in $[6,33,38]$ extensions of these criteria and applied them to similar questions. He further studied in $[34,36,37]$ the geometry of the sets $C_{f}$ defined in $(3.1)$, as well as their natural extension $C(A)=\left\{S_{n}(f), n \geq 1, f \in A\right\}$, in which $A$ is an arbitrary subset of $L^{2}(\mu)$. We also refer to Talagrand [31], where this question was investigated in a larger context.

Introduce the following commutation condition:

(C) There exists a sequence $\left\{T_{j}, j \geq 1\right\}$ of $L^{1}(\mu)$ positive isometries such that $T_{j} 1=1$, they commute with $S, S_{n}\left(T_{j} f\right)=T_{j}\left(S_{n} f\right)$, and

$$
\lim _{J \rightarrow \infty}\left\|\frac{1}{J} \sum_{j \leq J} T_{j} f-\int f d \mu\right\|_{1, \mu}=0
$$

for any $f \in L^{1}(\mu)$. Set

$$
C_{f}=\left\{S_{n}(f), n \geq 1\right\}
$$

for any $f \in L^{2}(\mu)$. Consider for $2 \leq p \leq \infty$ the following convergence property:

$$
\mu\left\{\left\{S_{n}(f), n \geq 1\right\} \text { converges }\right\}=1 \quad \text { for all } f \in L^{p}(\mu) .
$$

Bourgain's first criterion [2, Proposition 1] shows that if $\left(\mathcal{C}_{p}\right)$ holds for some $2 \leq p<\infty$, the sets $C_{f}$ cannot be too large. More precisely, the following holds.

Theorem 3.1. Let $S$ be a sequence of $L^{2}(\mu)$ contractions satisfying condition (C). Assume that $\left(\mathcal{C}_{p}\right)$ holds for some $2 \leq p<\infty$. Then there exists a numerical constant $C_{0}$ such that

$$
\sup _{\varepsilon>0} \varepsilon \sqrt{\log N_{f}(\varepsilon)} \leq C_{0}\|f\|_{2}
$$

for any $f \in L^{p}(\mu)$, where for any $\varepsilon>0, N_{f}(\varepsilon)$ denotes the minimal number of $L^{2}(\mu)$ open balls of radius $\varepsilon$ centered in $C_{f}$ and enough to cover $C_{f}$.

Remark 3.2. By using covering properties of ellipsoids, one can show that the above entropy estimate is optimal for convolutions on the circle and thus, it admits no improvement. See [33, p. 47]. However, it can be far from optimal on typical examples. Let $S_{n} f=\frac{1}{n} \sum_{j \leq n} T^{j} f$, where $T$ is some measure preserving transformation on $(X, \mathcal{A}, \mu)$. By a theorem of Talagrand, $N_{f}(\varepsilon) \leq C \max \left(1,\|f\|_{2, \mu}^{2} / \varepsilon^{2}\right), 0<\varepsilon \leq\|f\|_{2, \mu}$, where $C$ is an absolute constant. See [31] and $[32$, Theorem 1.4.1].

Bourgain's second criterion [2, Proposition 2] states as follows.

Theorem 3.3. Let $S$ be a sequence of $L^{2}(\mu)$ contractions satisfying condition (C). Assume that $\left(\mathcal{C}_{\infty}\right)$ is fulfilled. Then

$$
C(\delta)=\sup _{f \in L^{\infty}(\mu),\|f\|_{2} \leq 1} N_{f}(\delta)<\infty
$$


for any real $\delta>0$.

A starting point of the proof is a version (see [2, (9)]) of the Banach principle for $L^{\infty}(\mu)$, namely, the fact that the convergence property $\left(\mathcal{C}_{\infty}\right)$ implies that

$$
\sup _{\|f\|_{\infty, \mu} \leq 1,\|f\|_{2, \mu} \leq \varepsilon} \int_{X} \frac{S^{*} f}{1+S^{*} f} d \mu \rightarrow 0 \quad \text { as } \varepsilon \rightarrow 0 .
$$

This result was established soon after by Bellow and Jones in [4]. The proof is, however, lenghty and indirect. It is possible to provide a direct and short proof, similar to the one of the standard Banach principle, see [32, Theorem 5.1.5].

Note that the integrability of $S^{*} f$, which is required in $(3.2)$, is not ensured by the assumption made in Theorem 3.3. It is, for instance, guaranteed when $S_{n}$ are $L^{2}(\mu)-L^{\infty}(\mu)$ contractions, which is the case of all applications given in [2]. Moreover, Bourgain's proof runs with no modification using (3.2) at the conclusion.

Given a separable Hilbert space $H$, recall that the canonical Gaussian (also called isonormal) process $Z=\left\{Z_{h}, h \in H\right\}$ on $H$ is the centered Gaussian process with covariance function

$$
\Gamma\left(h, h^{\prime}\right)=\left\langle h, h^{\prime}\right\rangle, \quad h, h^{\prime} \in H .
$$

Let $\left\{h_{n}, n \geq 1\right\}$ be a countable orthonormal basis of $H$. Let also $\left\{g_{n}, n \geq 1\right\}$ be a sequence of i.i.d. $\mathcal{N}(0,1)$ distributed random variables on a basic probability space $(\Omega, \mathcal{A}, \mathbf{P})$. Then $Z$ can be defined as follows: for any $h \in H$,

$$
Z_{h}=\sum_{n=1}^{\infty} g_{n}\left\langle h, h_{n}\right\rangle .
$$

A subset $A$ of $H$ is a GB set (for Gaussian bounded) if the restriction of $Z$ on $A$ possesses a version that is sample bounded. Further, $A$ is a GC set (for Gaussian continuous) if the restriction of $Z$ on $A$ possesses a version that is sample $\|\cdot\|$-continuous. These notions were introduced in Dudley [9].

A countable subset $A$ of $H$ is a GB set if $\mathbf{E} \sup _{h \in A}|Z(h)|<\infty$, or, equivalently, $\mathbf{E} \sup _{h \in A} Z(h)<\infty$, since as is well-known,

$$
\mathbf{E} \sup _{h \in A} Z(h) \leq \mathbf{E} \sup _{h \in A}|Z(h)| \leq 2 \mathbf{E} \sup _{h \in A} Z(h)+\inf _{h_{0} \in A} \mathbf{E}\left|Z\left(h_{0}\right)\right| .
$$

Under assumptions of Theorem 3.1, Bourgain has also shown that the sets $C_{f}$ are GB sets. Some remarks are in order. It is not necessary to assume that $S_{n}$ are $L^{2}(\mu)$-contractions. Moreover, the conclusion remains true under a weaker condition than $\left(\mathcal{C}_{p}\right)$. Theorem 3.1 can be reformulated as follows.

Theorem 3.4. Let $S$ satisfy assumption (C). Assume that for some $2 \leq p<\infty$,

$$
\mu\left\{\sup _{n \geq 1}\left|S_{n}(f)\right|<\infty\right\}=1 \quad \text { for all } f \in L^{p}(\mu) .
$$

Then for any $f \in L^{p}(\mu)$, the sets $C_{f}$ are $G B$ sets of $L^{2}(\mu)$. Further, there exists a numerical constant $C_{1}$ and a constant $C_{2}$ such that

$$
C_{1} \sup _{\varepsilon>0} \varepsilon \sqrt{\log N_{f}(\varepsilon)} \leq \mathbf{E} \sup _{n \geq 1} Z\left(S_{n}(f)\right) \leq C_{2}\|f\|_{2, \mu}
$$

for any $f \in L^{p}(\mu)$.

The use of the fact that if $N(X)$ is a Gaussian semi-norm, then

$$
\mathbf{P}\{N(X) \leq s\}>0 \quad \Rightarrow \quad \mathbf{E} N(X) \leq \frac{4 s}{\mathbf{P}\{N(X) \leq s\}}
$$


slightly simplifies the proof, which otherwise is very similar (see [32]).

Remark 3.5. One can naturally wonder whether property $\left(\mathcal{C}_{p}\right)$ analogously implies that the sets $C_{f}$ are GC sets. This question was investigated in [33, Sec. 5.2.2], where in Theorem 5.2.4, it is shown that the answer is positive when $X=\mathbb{T}$ and $S_{n}$ are commuting with rotations.

Note before continuing that when $\int_{X} S^{*} f d \mu$ is finite, no explicit link with

$$
\mathbf{E} \sup _{n \geq 1} Z\left(S_{n}(f)\right)
$$

can be drawn from Theorem 3.4. In Theorem 3.6 below, this is established. A general inequality that is valid for arbitrary partial maxima can be directly indeed derived from condition (C) only. Let us add further comments. First, say a few words on the way in which the commutation condition $(C) \operatorname{links} Z$ and $S$. This explains easily. Let $f \in L^{2}(\mu)$ and let $I$ be a finite set of integers. Then one derives from $(C)$ that there exists an index $\mathcal{J}$ such that the two-sided inequalities

$$
\frac{1}{2}\left\|S_{n}(f)-S_{m}(f)\right\|_{2, \mu} \leq\left\|S_{n}\left(F_{J, f}\right)-S_{m}\left(F_{J, f}\right)(x)\right\|_{2, \mathbf{P}} \leq 2\left\|S_{n}(f)-S_{m}(f)\right\|_{2, \mu}
$$

hold true for all $n, m \in I$ and all $J \in \mathcal{J}$, and for all $x$ in a measurable set of positive measure. See Lemma 4.6. Theorem 3.1 is obtained as a straightforward application of the Banach principle and Slepian's inequality combined with Sudakov's minoration (Lemma 4.9).

Bourgain essentially applied Theorem 3.3, and this in the case where $X=\mathbb{T}$ and $T_{j}$ are translation or dilation operators. The counter-examples are built on functions of the type

$$
f=\frac{1}{\sqrt{\#(F)}} \sum_{n \in F} e_{n}, \quad e_{n}(x)=e^{2 i \pi n x},
$$

where $F$ are specific arithmetic sets. These elements, as well as all $T_{j} f, j \geq 1$, not only belong to $L^{p}(\mu)$ but also to many more specific spaces. So that for Banach spaces $B$ such that $B \subset L^{2}(\mu)$, a requirement on $f \in B$ like

$$
T_{j} f \in B, \quad j \geq 1,
$$

is frequently non void. Call $\mathcal{R}(B)$ the set of these elements. Then $F_{J, f} \in B$ whenever $f \in$ $\mathcal{R}(B)$. If, for instance, $B=L^{p}(\mu)$, then by Corollary 4.3 and Lemma $4.4, \mathcal{R}(B)=B$.

Theorem 3.6. Let $S$ satisfy condition (C). Let, additionally, I be a finite set of integers and $0<\varepsilon<1$. Then there exists a partial index $\mathcal{J}$ such that for any $J \in \mathcal{J}$ and any positive increasing convex function $G: \mathbb{R}^{+} \rightarrow \mathbb{R}^{+}$, the following statement are true.

(i) Let $B \subset L^{2}(\mu)$ be a Banach space with norm $\|\cdot\|_{B}$. For any $f \in \mathcal{R}(B)$,

$$
\sqrt{1-\varepsilon} \mathbf{E} \sup _{n \in I} Z\left(S_{n}(f)\right) \leq \mathbf{E}\left\|F_{J, f}\right\|_{B} \sup _{\|h\|_{B} \leq 1} \int \sup _{n \in I}\left|S_{n}(h)\right| d \mu .
$$

Moreover,

$$
\begin{aligned}
\mathbf{E} G\left(\sqrt{1-\varepsilon} \sup _{n, m \in I}\left|Z\left(S_{n}(f)\right)-Z\left(S_{m}(f)\right)\right|\right) \\
\quad \leq \mathbf{E}\left\|F_{J, f}\right\|_{B} \sup _{\|h\|_{B} \leq 1} \mathbf{E} \int_{X} G\left(\sup _{n, m \in I}\left|\left(S_{n}-S_{m}\right)(h)\right|\right) d \mu .
\end{aligned}
$$


(ii) In particular,

$$
\sqrt{1-\varepsilon} \sup _{\|f\|_{2, \mu} \leq 1} \mathbf{E} \sup _{n \in I} Z\left(S_{n}(f)\right) \leq C_{p} \sup _{\|h\|_{p, \mu} \leq 1} \int \sup _{n \in I}\left|S_{n}(h)\right| d \mu
$$

for any $f \in L^{p}(\mu)$ with $2 \leq p<\infty$, where $C_{p}=\|g\|_{p} /\|g\|_{2}$, recalling the notation used. Further,

$$
\begin{aligned}
\sup _{\|f\|_{2, \mu} \leq 1} \mathbf{E} G\left(\sqrt{1-\varepsilon} \sup _{n, m \in I} \mid Z\left(S_{n}(f)\right)-\right. & \left.Z\left(S_{m}(f)\right) \mid\right) \\
& \leq C_{p} \sup _{\|h\|_{p, \mu} \leq 1} \mathbf{E} \int_{X} G\left(\sup _{n, m \in I}\left|\left(S_{n}-S_{m}\right)(h)\right|\right) d \mu .
\end{aligned}
$$

We have the following criterion providing a general form of Theorem 3.4.

Theorem 3.7. Let $S$ satisfy assumption (C). Let $B \subset L^{2}(\mu)$ be a Banach space with norm $\|\cdot\|_{B}$. Assume that the following property is fulfilled:

$$
\mu\left\{\sup _{n \geq 1}\left|S_{n}(f)\right|<\infty\right\}=1 \quad \text { for all } f \in B .
$$

Then there exists a constant $K$ depending on $S$ and $B$ only such that

$$
\mathbf{E} \sup _{n \geq 1} Z\left(S_{n}(f)\right) \leq K \limsup _{H \rightarrow \infty} \mathbf{E}\left\|F_{H, f}\right\|_{B} \quad \text { for all } f \in \mathcal{R}(B) .
$$

Let us derive a criterion which has been recently applied in [6] to show the optimality of a famous theorem of Koksma. Let $\left\{h_{n}, n \in \mathbb{Z}\right\}$ be a countable orthonormal basis of $L^{2}(\mu)$ and use the notation $f \sim \sum_{n \in \mathbb{Z}} a_{n}(f) h_{n}, \sum_{n \in \mathbb{Z}} a_{n}^{2}(f)<\infty$, if $f \in L^{2}(\mu)$. Given a sequence of positive reals $w=\left\{w_{n}, n \in \mathbb{Z}\right\}$ with $w_{n} \geq 1$, we recall that $L_{w}^{2}(\mu)$ is the subspace of $L^{2}(\mu)$ consisting of functions such that

$$
\sum_{n \in \mathbb{Z}} w_{n} a_{n}^{2}(f)<\infty
$$

This is a Hilbert space with scalar product defined by

$$
\langle f, h\rangle=\sum_{n \in \mathbb{Z}} w_{n} a_{n}(f) a_{n}(h)
$$

and norm

$$
\|f\|_{2, w}=\left(\sum_{n \in \mathbb{Z}} w_{n} a_{n}^{2}(f)\right)^{1 / 2} .
$$

The space $L^{2}(\mu)$ corresponds to the case $w_{n} \equiv 1$. Moreover, $L_{w}^{2}(\mu)$ trivially contains any $f$ such that $a_{n}(f)=0$ except for finitely many $n$.

Corollary 3.8. Let $S$ satisfy assumption (C). Assume that the following property is fulfilled:

$$
\mu\left\{\sup _{n \geq 1}\left|S_{n}(f)\right|<\infty\right\}=1 \quad \text { for all } f \in L_{w}^{2}(\mu) .
$$

Then there exists a constant $K$ depending on $S$ and $w$ only such that

$$
\sup _{\varepsilon>0} \varepsilon \sqrt{\log N_{f}(\varepsilon)} \leq K \limsup _{J \rightarrow \infty} \mathbf{E}\left\|F_{J, f}\right\|_{2, w} \quad \text { for all } f \in \mathcal{R}\left(L_{w}^{2}(\mu)\right) .
$$

Remark 3.9. Let $X=\mathbb{T}$, let $\mu$ be the normalized Lebesgue measure, and let $T_{j}$ be dilation operators, $T_{j} f(x)=f(j x)$. Then any finite trigonometric sum belongs to $\mathcal{R}\left(L_{w}^{2}(\mu)\right)$. 
We refer to [32, Chap. 6] for a study of the link between the partial maximum operators (where $I$ is a set of integers),

$$
\sup _{\substack{\|h\|_{\infty, \mu \leq 1} \leq\left\|^{\prime}\right\|_{2, \mu} \leq \varepsilon \\ \|}} \sup _{n \in I}\left|S_{n}(h)\right| d \mu \quad \text { and } \quad \sup _{\|f\|_{2, \mu} \leq 1} \mathbf{E} \sup _{n \in I} Z\left(S_{n}(f)\right) .
$$

In the theorem below, we provide a quantitative link.

Theorem 3.10. Let $S_{n}, n \geq 1$, be $L^{2}(\mu)-L^{\infty}(\mu)$ continuous operators verifying condition (C). Let $I$ be any set of integers with cardinality $M$. For any reals $A>0$ and $R>0$, it is true that

$$
\begin{aligned}
\sup _{\|f\|_{2, \mu} \leq 1} \mathbf{E} \sup _{n \in I} Z\left(S_{n}(f)\right) \leq 6 \sqrt{M} S_{1}(I) \exp \left\{-A^{2} / 8\right\} & +A(\sqrt{2}) S_{2}(I) e^{-R^{2} / 4} \\
& +A \sup _{\substack{\|h\|_{\infty, \mu \leq 1} \leq 1 \\
\|h\|_{2, \mu} \leq R / A}} \int_{\substack{X \\
\sup _{n \in I}}}\left|S_{n}(h)\right| d \mu,
\end{aligned}
$$

where $S_{1}(I)=\max _{n \in I}\left\|S_{n}\right\|_{2}, S_{2}(I)=\max _{n \in I}\left\|S_{n}\right\|_{\infty}$

$$
\left\|S_{n}\right\|_{2}=\sup _{\|f\|_{2} \leq 1}\left\|S_{n}(f)\right\|_{2}, \quad \text { and } \quad\left\|S_{n}\right\|_{\infty}=\sup _{\|f\|_{\infty} \leq 1}\left\|S_{n}(f)\right\|_{\infty} .
$$

Remark 3.11. It is not complicate to derive from this bound Theorem 3.3 for $L^{2}(\mu)-L^{\infty}(\mu)$ contractions.

Now consider the spaces $L^{p}(\mu), 1<p<2$. A corresponding entropy criterion can also be established.

Theorem 3.12. Let $1<p \leq 2$ with conjugate number $q$. Consider a sequence $S=\left\{S_{n}, n \geq 1\right\}$ of continuous operators from $L^{p}(\mu)$ to $L^{p}(\mu)$. Assume that condition (C) is satisfied.

Further assume that property $\left(\mathcal{B}_{r}\right)$ is satisfied for some real $0<r<p$. Then there exists a constant $C(r, p)<\infty$ depending on $r$ and $p$ only such that

$$
\sup _{\varepsilon>0} \varepsilon\left(\log N_{f}^{p}(\varepsilon)\right)^{1 / q} \leq C(r, p)\|f\|_{p}
$$

for any $f \in L^{p}(\mu)$, where $N_{f}^{p}(\varepsilon)$ is the minimal number of open $L^{p}$-balls of radius $\varepsilon$ centered in $C_{f}$ and enough to cover it. Further, $C(r, p)$ tends to infinity as $r$ tends to $p$.

The proof given in [33] relies on properties of $p$-stable processes; it is assumed that $S$ commutes with an ergodic endomorphism of $(X, \mathcal{A}, \mu)$, which, in fact, is unnecessary. The restriction $p \neq 1$ is only used at the very end of the proof, but is then crucially necessary.

Remark 3.13. The pending question of a possible convergence criterion for the space $L^{1}(\mu)$ is, of course, very interesting. But its true nature is unknown, since we are not operating in a (strictly) stationary context. In particular, $\left\|S_{n}(f)-S_{m}(f)\right\|_{p, \mu}$, crucial in (5.11), does not even depend on $n-m$ only, in general. Moreover, we know (see Talagrand [30, Sec. 8.1]) that a necessary condition for a 1-stable process to be sample bounded rather expresses in terms of majorizing measures. This important concept is, however, not relevant in the present context because of its difficulty of application.

As announced already, we have made the paper self-contained. We provide proofs of these theorems in Sec. 5. 


\section{Auxiliary Results}

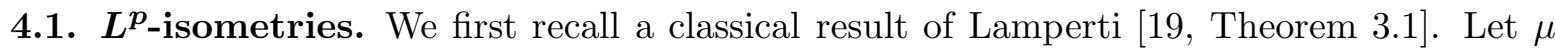
be a $\sigma$-finite measure on $(X, \mathcal{A})$. Some basic properties of isometries of $L^{p}(\mu)$ are used in what follows. Recall that a regular set-isomorphism of the measure space $(X, \mathcal{A}, \mu)$ is a mapping $\Theta$ of $\mathcal{A}$ into itself such that

$$
\begin{aligned}
& \Theta\left(A^{c}\right)=\Theta X \backslash \Theta A ; \\
& \Theta\left(\bigcup_{n=1}^{\infty} A_{n}\right)=\bigcup_{n=1}^{\infty} \Theta A_{n} \text { for disjoint } A_{n} ; \\
& \mu(\Theta A)=0 \text { if and only if } \mu(A)=0,
\end{aligned}
$$

for all elements $A, A_{n}$ of $\mathcal{A}$. Then $\Theta$ induces a linear transformation (denoted again by $\Theta$ ) on the space of measurable functions defined as follows: $\Theta \chi_{A}=\chi_{\Theta A}$.

Remark 4.1. The question whether a measure preserving set transformation can be obtained from a point mapping has been already considered. By a result of von Neumann, so is the case if, for instance, $X$ is a closed region in $\mathbb{R}^{n}$ and $\mu$ is equivalent to the Lebesgue measure, see $[19$, p. 463].

Lemma 4.2. Let $T$ be a linear operator on $L^{p}(\mu)$, where $1 \leq p<\infty$ and $p \neq 2$, such that $\|T f\|_{p}=\|f\|_{p}$ for all $f \in L^{p}(\mu)$. Then there exists a regular set-isomorphism $\Theta$ and a function $h(x)$ such that $T$ is given by

$$
T f(x)=h(x) \Theta f(x) .
$$

Define a measure $\mu^{*}$ by $\mu^{*}(A)=\mu\left(\Theta^{-1} A\right)$. Then

$$
|h(x)|^{p}=\frac{d \mu^{*}}{d \mu}(x) \quad \text { a.e. on } \Theta X .
$$

Corollary 4.3. Let $\mu$ be a probability measure. Let $T$ be a positive isometry of $L^{p}(\mu)$ with $1 \leq p<\infty$ and $p \neq 2$ such that $T 1=1$. Then $T f(x)=\Theta f(x)$ with $\Theta 1=1$, and $\Theta$ is a regular set-isomorphism. Moreover, for any $f \in L^{\infty}(\mu),|T f|^{a} \stackrel{\text { a.e. }}{=} T|f|^{a}$ for any $0 \leq a<\infty$. Further, $|T f|^{p} \stackrel{\text { a.e. }}{=} T|f|^{p}$ if $f \in L^{p}(\mu)$.

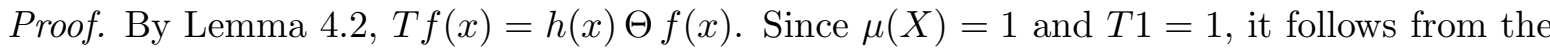
proof of Theorem 3.1 in [19] that $h(x) \stackrel{\text { a.e. }}{=} 1$, and $T=\Theta$. But since $\Theta \chi_{A}=\chi_{\Theta A}$, we get the equality $|T f|^{a}=T|f|^{a}$ for simple functions for any $0 \leq a<\infty$. Hence, by approximation, the equality $|T f|^{a} \stackrel{\text { a.e. }}{=} T|f|^{a}$ holds for all $f \in L^{\infty}(\mu)$. Further, by approximation again, since $T$ is an isometry of $L^{p}(\mu),|T f|^{p} \stackrel{\text { a.e. }}{=} T|f|^{p}$ if $f \in L^{p}(\mu)$.

For the sake of completeness, we included the following lemma concerning the (simpler) case $p=2$.

Lemma 4.4. Let $T$ be a positive isometry of $L^{2}(\mu)$ such that $T 1=1$. Then $(T f)^{2} \stackrel{\text { a.e. }}{=} T f^{2}$ for any $f \in L^{2}(\mu)$.

Proof. Let $A \in \mathcal{A}$ with $0<\mu(A)<1$. Trivially, $f, g \in L_{+}^{2}(\mu)$ have disjoint supports if and only if $\|f+g\|_{2, \mu}^{2}=\|f\|_{2, \mu}^{2}+\|g\|_{2, \mu}^{2}$. Hence, it follows that $T \mathbf{1}_{A}$ and $T \mathbf{1}_{A^{c}}$ have disjoint supports. Let $E=\left\{0<T \mathbf{1}_{A}<1\right\}=\left\{0<T \mathbf{1}_{A^{c}}<1\right\}$. Since $E \subset \operatorname{supp}\left(T \mathbf{1}_{A}\right) \cap \operatorname{supp}\left(T \mathbf{1}_{A^{c}}\right)$, we conclude that $T \mathbf{1}_{A}$ and $T \mathbf{1}_{A^{c}}$ are indicator functions. Thus, any simple function is mapped by $T$ to a simple function. For these functions, $(T f)^{2}=T f^{2}$. Now let $f \in L_{+}^{2}(\mu)$; there exists a sequence 
$\left(f_{n}\right) \subset L^{\infty}(\mu)$ such that $\left\|f-f_{n}\right\|_{2} \rightarrow 0$ as $n \rightarrow \infty$. First observe by applying twice Hölder's inequality that

$$
\int_{X}\left(T\left|f_{n}^{2}-f^{2}\right|\right)^{1 / 2} d \mu \leq\left(\int_{X} T\left|f_{n}^{2}-f^{2}\right| d \mu\right)^{1 / 2}=\left\|f_{n}^{2}-f^{2}\right\|_{1}^{1 / 2} \leq\left(\left\|f_{n}-f\right\|_{2} \cdot\left\|f_{n}+f\right\|_{2}\right)^{1 / 2} .
$$

Consequently,

$$
\begin{aligned}
& \left\|T f-\sqrt{T f^{2}}\right\|_{1} \leq\left\|T f-T f_{n}\right\|_{1}+\left\|T f_{n}-\sqrt{T f_{n}^{2}}\right\|_{1}+\left\|\sqrt{T f_{n}^{2}}-\sqrt{T f^{2}}\right\|_{1} \\
& \quad=\left\|f-f_{n}\right\|_{1}+\left\|\sqrt{T f_{n}^{2}}-\sqrt{T f^{2}}\right\|_{1} \leq\left\|f-f_{n}\right\|_{1}+\left(\left\|f_{n}-f\right\|_{2} \cdot\left\|f_{n}+f\right\|_{2}\right)^{1 / 2} \rightarrow 0
\end{aligned}
$$

as $n \rightarrow \infty$. Hence, $(T f)^{2}=f^{2}$ a.e. Since $f=f^{+}-f^{-}$, we deduce that this holds for any $f \in L^{2}(\mu)$.

4.2. Stable processes. This part was essentially written for the ergodician reader not necessarily familiar with stable processes. We use very few from the theory. We refer to [24]. We also refer the interested reader to the very nice book of Talagrand [30] for a thorough study of regularity of stable processes. For the same reason, the last part of the proof of Theorem 3.12 is detailed, and we refer to [24]. The stable processes we consider are simple, of finite rank. They are, however, not strongly stationary. Recall and briefly explain some basic facts and properties of stable random variables and stable processes.

Let $0<\alpha \leq 2$. A real-valued random variable $\theta$ is symmetric $\alpha$-stable of parameter $\sigma$ if

$$
\mathbf{E} e^{i t \theta}=e^{-\sigma^{\alpha}|t|^{\alpha}} \quad \text { for any } \quad t \in \mathbb{R} .
$$

Then for all $0<r<\alpha,\left(\mathbf{E}|\theta|^{r}\right)^{1 / r}=\delta(r, \alpha) \sigma$, where $\delta(r, \alpha)$ depends only on $r$ and $\alpha$. Stable random variables are mixtures of Gaussian random variables. Indeed, as is well known, the function $f(\lambda)=e^{-\lambda^{\alpha}}$ is completely monotone on $\mathbb{R}^{+}$for each $0<\alpha \leq 1$. Consequently, there exists a random variable $v(\alpha)$ such that $\mathbf{E} e^{-\lambda v(\alpha)}=f(\lambda)$ for all $\lambda \geq 0$. Let $\eta(\alpha):=$ $(2 v(\alpha / 2))^{1 / 2}$. Let $g$ be standard Gaussian independent from $\eta$. By taking Fourier transforms, $\mathbf{E} e^{i t \eta(\alpha) \cdot g}=\mathbf{E} e^{-t^{2} \eta(\alpha)^{2} / 2}=\mathbf{E} e^{-t^{2} v(\alpha / 2)}=e^{-|t|^{\alpha}}$. Whence it follows that $\theta \stackrel{\mathcal{D}}{=} \eta(\alpha) \cdot g$. Let $\theta_{1}, \ldots, \theta_{J}$ be i.i.d. $\alpha$-stable real-valued random variables and let $c_{1}, \ldots, c_{J}$ be real numbers. From (4.2) we get the equality

$$
\sum_{j=1}^{J} c_{j} \theta_{j} \stackrel{\mathcal{D}}{=} \theta_{1}\left(\sum_{j=1}^{J}\left|c_{j}\right|^{\alpha}\right)^{1 / \alpha}
$$

A stochastic process $\{X(t), t \in T\}$ is real-valued $\alpha$-stable if any finite linear combination $\sum_{j} c_{j} X\left(t_{j}\right)$ is an $\alpha$-stable real-valued random variable.

From now on, we extend the notation used in (2.4) in the following way. To any $f \in L^{p}(\mu)$, $1<p \leq \infty$, we associate the random element

$$
F_{J, f}(\omega, x)=\frac{1}{J^{1 / p}} \sum_{1 \leq j \leq J} \theta_{j}(\omega) T_{j} f(x), \quad \omega \in \Omega, x \in X .
$$

Remark 4.5. As long as entropy criteria are studied in $L^{p}(\mu)$ with $2 \leq p \leq \infty$, the relevant random elements $F_{J, f}$ are Gaussian $(\alpha=2)$. When $1<p<2$, we choose them $p$-stable $(\alpha=p)$. 
Clearly, (4.4) defines a real-valued $\alpha$-stable process. It follows, in particular, that

$$
F_{J, f}(\cdot, x) \stackrel{\mathcal{D}}{=} \theta_{1}\left(\frac{1}{J} \sum_{1 \leq j \leq J}\left|T_{j} f(x)\right|^{\alpha}\right)^{1 / \alpha}
$$

for any $x \in X$. Let $\left\{\eta_{j}, j=1, \ldots, J\right\}$ be a sequence of i.i.d. random variables with the same law as $\eta(\alpha)$ and let $\left\{g_{j}, j=1, \ldots, J\right\}$ be a sequence of i.i.d. Gaussian standard random variables. We assume that these sequences are defined on joint probability spaces $\left(\Omega^{\prime}, \mathcal{B}^{\prime}, \mathbf{P}^{\prime}\right)$ and $\left(\Omega^{\prime \prime}, \mathcal{B}^{\prime \prime}, \mathbf{P}^{\prime \prime}\right)$, respectively.

Then the process

$$
\mathcal{F}_{J, f}\left(\omega^{\prime}, \omega^{\prime \prime}, x\right)=\sum_{j \leq J} \eta_{j}\left(\omega^{\prime}\right) g_{j}\left(\omega^{\prime \prime}\right) T_{j} f(x), \quad x \in X,
$$

has the same distribution as $\left\{F_{J, f}(x), x \in X\right\}$.

4.3. A comparison lemma. In the next lemma, we denote the norms corresponding to the spaces $L^{r}(\mu)$ and $L^{r}(\mathbf{P})$ by $\|\cdot\|_{r, \mu}$ and $\|\cdot\|_{r, \mathbf{P}}$, respectively.

Lemma 4.6. Let $1 \leq p \leq 2$. Let $S_{n}: L^{p}(\mu) \rightarrow L^{p}(\mu), n=1,2, \ldots$, be continuous operators verifying assumption $(\mathrm{C})$.

(i) Let $1 \leq p<2$. Let $f \in L^{p}(\mu)$ and $0<\varepsilon<1$. Let also $I$ be a finite set of integers such that

$$
\left\|S_{n}(f)-S_{m}(f)\right\|_{p, \mu} \neq 0 \quad \text { for all } n \neq m, n, m \in I .
$$

Then given any index $\mathcal{J}_{0}$, there exists a sub-index $\mathcal{J}$ and a measurable set $A$ with $\mu\{A\} \geq 1-\varepsilon$ and such that

$$
(1-\varepsilon)^{1 / p} \leq \frac{\left\|\left(S_{n}-S_{m}\right)\left(F_{J, f}\right)(x)\right\|_{r, \mathbf{P}}}{c(r)\left\|S_{n}(f)-S_{m}(f)\right\|_{p, \mu}} \leq(1+\varepsilon)^{1 / p}
$$

for all $x \in A$, for all $J \in \mathcal{J}$, all $n, m \in I, m \neq n$, and for $r<p$. Moreover, $c(r)=\left\|\theta_{1}\right\|_{r}$.

(ii) Let $p=2$. In place of (4.6), we have the inequalities

$$
(1-\varepsilon)^{1 / 2} \leq \frac{\left\|\left(S_{n}-S_{m}\right)\left(F_{J, f}\right)(x)\right\|_{2, \mathbf{P}}}{\left\|S_{n}(f)-S_{m}(f)\right\|_{2, \mu}} \leq(1+\varepsilon)^{1 / 2}
$$

for all $J \in \mathcal{J}$ and all $n, m \in I, m \neq n$. Further, for any positive increasing convex function $G$ on $\mathbb{R}^{+}$and any $J \in \mathcal{J}$,

$$
\mathbf{E} G\left(\sqrt{1-\varepsilon} \sup _{n, m \in I} Z\left(S_{n}(f)\right)-Z\left(S_{m}(f)\right)\right) \leq \mathbf{E} \int_{X} G\left(\sup _{n, m \in I} S_{n}\left(F_{J, f}\right)-S_{m}\left(F_{J, f}\right)\right) d \mu .
$$

In particular, for any $J \in \mathcal{J}$,

$$
\sqrt{1-\varepsilon} \mathbf{E} \sup _{n \in I} Z\left(S_{n}(f)\right) \leq \mathbf{E} \int_{X} \sup _{n \in I} S_{n}\left(F_{J, f}\right) d \mu .
$$

Proof. We assume that $\mathcal{J}_{0}=\mathbb{N}$; the case of an arbitrary index $\mathcal{J}_{0}$ is treated identically.

Proof of (i). Let $f \in L^{p}(\mu)$. By the commutation assumption,

$$
S_{n}\left(F_{J, f}\right)=\frac{1}{J^{\frac{1}{p}}} \sum_{j \leq J} \theta_{j} S_{n}\left(T_{j}(f)\right)=\frac{1}{J^{1 / p}} \sum_{j \leq J} \theta_{j} T_{j}\left(S_{n}(f)\right) \text { for all } n \geq 1, J \geq 1 .
$$

Hence, by (4.3),

$$
\frac{1}{J^{\frac{1}{p}}} \sum_{j \leq J} \theta_{j} T_{j}\left(S_{n}(f)-S_{m}(f)\right)(x) \stackrel{\mathcal{D}}{=} \theta_{1}\left(\frac{1}{J} \sum_{j \leq J}\left|T_{j}\left(S_{n}(f)-S_{m}(f)\right)(x)\right|^{p}\right)^{1 / p}
$$


for any fixed $x \in X$. Using the fact that $\left|T_{j} h\right|^{p} \stackrel{\text { a.e. }}{=} T_{j}|h|^{p}$ if $h \in L^{p}(\mu)$, it follows that

$$
\begin{aligned}
\mathbf{E}\left|\left(S_{n}-S_{m}\right)\left(F_{J, f}\right)(x)\right|^{r} & =\left(\mathbf{E}\left|\theta_{1}\right|^{r}\right)\left(\frac{1}{J} \sum_{j \leq J}\left|T_{j}\left(S_{n}(f)-S_{m}(f)\right)(x)\right|^{p}\right)^{r / p} \\
& =\left(\mathbf{E}\left|\theta_{1}\right|^{r}\right)\left(\frac{1}{J} \sum_{j \leq J} T_{j}\left(\left|S_{n}(f)-S_{m}(f)\right|^{p}\right)(x)\right)^{r / p}
\end{aligned}
$$

for almost all $x$.

Let $I$ be a finite set of integers such that

$$
\left\|S_{n}(f)-S_{m}(f)\right\|_{p, \mu} \geq \delta>0 \quad \text { for all } n \neq m, n, m \in I .
$$

Let $0<\varepsilon<1$ and choose an integer $L$ sufficiently large so that $2^{-L} \leq \varepsilon$ and $\delta \geq 2^{-L-1} / \varepsilon$. Assumption (C) implies that

$$
\lim _{J \rightarrow \infty}\left\|\frac{1}{J} \sum_{j \leq J} T_{j}\left(\left|S_{n}(f)-S_{m}(f)\right|^{p}\right)-\right\| S_{n}(f)-S_{m}(f)\left\|_{p, \mu}^{p}\right\|_{1, \mu}=0
$$

for all $n, m \in I$. By extraction, we can find an index $\mathcal{J}=\left\{J_{k}, k>L\right\}$ depending on $I$ and $\varepsilon$ such that

$$
\left\|\frac{1}{J_{k}} \sum_{j \leq J_{k}} T_{j}\left(\left|S_{n}(f)-S_{m}(f)\right|^{p}\right)-\right\| S_{n}(f)-S_{m}(f)\left\|_{p, \mu}^{p}\right\|_{1, \mu} \leq \frac{1}{\#(I)^{2} 2^{2 k}}
$$

for all $n, m \in I$ and all $k>L$. Put

$$
\begin{aligned}
& A_{k}=\{\text { there exist } n, m \in I \quad \text { such that } \\
&\left.\qquad\left|\frac{1}{J_{k}} \sum_{j \leq J_{k}} T_{j}\left(\left|S_{n}(f)-S_{m}(f)\right|^{p}\right)-\left\|S_{n}(f)-S_{m}(f)\right\|_{p, \mu}^{p}\right| \geq 2^{-k}\right\}, \quad k>L .
\end{aligned}
$$

By Chebyshev's inequality, $\mu\left(A_{k}\right) \leq 2^{-k}$. Let

$$
\begin{aligned}
A_{\varepsilon}(n, m, J)=\left\{(1-\varepsilon)\left\|S_{n}(f)-S_{m}(f)\right\|_{p, \mu}^{p}\right. & \leq \frac{1}{J} \sum_{j \leq J} T_{j}\left(\left|S_{n}(f)-S_{m}(f)\right|^{p}\right) \\
& \left.\leq(1+\varepsilon)\left\|S_{n}(f)-S_{m}(f)\right\|_{p, \mu}^{p}\right\}
\end{aligned}
$$

and

$$
A_{I, \varepsilon}=\bigcap_{k>L} \bigcap_{n, m \in I} A_{\varepsilon}\left(n, m, J_{k}\right)
$$

Then

$$
\mu\left\{A_{I, \varepsilon}\right\} \geq \mu\left\{\bigcap_{k>L} A_{k}^{c}\right\} \geq 1-\sum_{k>L} 2^{-k}=1-2^{-L} \geq 1-\varepsilon
$$

Since

$$
\left\|\left(S_{n}-S_{m}\right)\left(F_{J, f}\right)(x)\right\|_{r, \mathbf{P}}=\left\|\theta_{1}\right\|_{r}\left(\frac{1}{J} \sum_{j \leq J} T_{j}\left(\left|S_{n}(f)-S_{m}(f)\right|^{p}\right)(x)\right)^{1 / p}
$$

for any $r<p$ by (4.9), it follows that

$$
(1-\varepsilon)^{1 / p} \leq \frac{\left\|\left(S_{n}-S_{m}\right)\left(F_{J, f}\right)(x)\right\|_{r, \mathbf{P}}}{\left\|\theta_{1}\right\|_{r}\left\|S_{n}(f)-S_{m}(f)\right\|_{p, \mu}} \leq(1+\varepsilon)^{1 / p}
$$

for every $x \in A_{I, \varepsilon}$, for all $J \in \mathcal{J}$, all $n, m \in I, m \neq n$, and $r<p$. 
Proof of (ii). The proof of the first inequality is identical, and so we omit it. Let $f \in L^{2}(\mu)$. Let $0<\varepsilon<1$ be fixed. Let $I$ be a finite set of integers such that

$$
\left\|S_{n}(f)-S_{m}(f)\right\|_{p, \mu} \neq 0 \quad \text { for all } n \neq m, n, m \in I .
$$

Now notice that $\mu\left\{\mathbf{E} \sup _{n \in I} S_{n}\left(F_{J, f}\right) \geq 0\right\}=1$. Using (4.7) and next the Slepian comparison lemma, we have along the index $\mathcal{J}$,

$$
\begin{aligned}
\int_{X} \mathbf{E} \sup _{n \in I} S_{n}\left(F_{J, f}\right) d \mu & \geq \int_{A} \mathbf{E} \sup _{n \in I} S_{n}\left(F_{J, f}\right) d \mu \\
& \geq \sqrt{1-\varepsilon} \mu(A) \mathbf{E} \sup _{n \in I} Z\left(S_{n}(f)\right) \geq(1-\varepsilon) \mathbf{E} \sup _{n \in I} Z\left(S_{n}(f)\right) .
\end{aligned}
$$

Similarly,

$$
\begin{aligned}
\int_{X} \mathbf{E} G\left(\sup _{n, m \in I} S_{n}\left(F_{J, f}\right)-S_{m}\left(F_{J, f}\right)\right) d \mu & \geq \int_{A} \mathbf{E} G\left(\sup _{n, m \in I} S_{n}\left(F_{J, f}\right)-S_{m}\left(F_{J, f}\right)\right) d \mu \\
& \geq \int_{A} \mathbf{E} G\left(\sqrt{1-\varepsilon} \sup _{n, m \in I} Z\left(S_{n}(f)\right)-Z\left(S_{m}(f)\right)\right) d \mu \\
& \geq \sqrt{1-\varepsilon} \mathbf{E} G\left(\sqrt{1-\varepsilon} \sup _{n, m \in I} Z\left(S_{n}(f)\right)-Z\left(S_{m}(f)\right)\right) \\
& \geq \sqrt{1-\varepsilon} \mathbf{E} G\left(\sqrt{1-\varepsilon} \sup _{n, m \in I} Z\left(S_{n}(f)\right)-Z\left(S_{m}(f)\right)\right) .
\end{aligned}
$$

This completes the proof of Lemma 4.6.

4.4. Banach Principle. Let

$$
\mathcal{Y}=\left\{f \in L^{\infty}(\mu):\|f\|_{\infty} \leq 1\right\} .
$$

A mapping $V:(\mathcal{Y}, d) \rightarrow L^{0}(\mu)$ is said to be continuous at 0 if $V$ is $d$-continuous at 0 on $\mathcal{Y}$. When $V$ is linear, then $V$ is continuous at 0 if and only if $V$ is $d$-continuous on $L^{\infty}(\mu)$.

Lemma 4.7 ([4]). Let $\left\{S_{n}, n \geq 1\right\}$ be a sequence of linear operators of $L^{\infty}(\mu)$ in $L^{0}(\mu)$. Assume that the following conditions are realized:

(i) Each $S_{n}$ is continuous at 0 ;

(ii) for any $f \in L^{\infty}(\mu), \mu\left\{x:\left\{S_{n}(f)(x), n \geq 1\right\}\right.$ converges $\}=1$.

Then $S^{*}: \mathcal{Y} \rightarrow L^{0}(\mu)$ is continuous at 0 .

For a short proof, we refer to [32, p. 205]. The next lemma is used repeatedly.

Lemma 4.8. Let $\left(B,\|\cdot\|_{B}\right)$ be a Banach space and let $S_{n}: B \rightarrow L^{0}(\mu), n \geq 1$, be continuous in measure operators. Assume that

$$
\mu\left\{\sup _{n \geq 1}\left|S_{n}(f)\right|<\infty\right\}=1 \quad \text { for all } f \in B .
$$

Then there exists a nonincreasing function $C:[0,1] \rightarrow \mathbb{R}_{+}$such that for any $0<\varepsilon<1$, any $J \geq 1$, and any $f \in \mathcal{R}(B)$, there exists a measurable set $X_{\varepsilon, J, f}$ with $\mu\left(X_{\varepsilon, J, f}\right) \geq 1-\varepsilon$ such that

$$
\mathbf{P}\left\{\omega: \sup _{n \geq 1}\left|S_{n}\left(F_{J, f}(\omega, \cdot)\right)(x)\right| \leq C(\varepsilon) \mathbf{E}\left\|F_{J, f}\right\|_{B}\right\} \geq 1-\varepsilon
$$

for any $x \in X_{\varepsilon, J, f}$, recalling that $F_{J, f}$ are defined in (4.4). 
Proof. By the Banach principle, there exists a nonincreasing function $\delta:[0,1] \rightarrow \mathbb{R}_{+}$such that

$$
\mu\left\{\sup _{n}\left|S_{n}(h)\right| \geq \delta(\varepsilon)\|h\|_{B}\right\} \leq \varepsilon^{2} / 2 \quad \text { for any } \quad 0<\varepsilon \leq 1 \quad \text { and } \quad h \in B .
$$

If $f \in \mathcal{R}(B)$, then $F_{J, f} \in \mathcal{R}(B)$ almost surely. Taking $h=F_{J, f}$ and using Fubini's theorem, we get the inequality

$$
\int_{X} \mathbf{P}\left\{\sup _{n \geq 1}\left|S_{n}\left(F_{J, f}\right)\right| \geq \delta(\varepsilon)\left\|F_{J, f}\right\|_{B}\right\} d \mu \leq \varepsilon^{2} / 2 .
$$

Now we bound as follows:

$$
\begin{aligned}
& \int_{X} \mathbf{P}\left\{\sup _{n \geq 1}\left|S_{n}\left(F_{J, f}\right)\right| \geq \frac{2 \delta(\varepsilon)}{\varepsilon^{2}} \mathbf{E}\left\|F_{J, f}\right\|_{B}\right\} d \mu \\
& \leq \int_{X} \mathbf{P}\left\{\sup _{n \geq 1}\left|S_{n}\left(F_{J, f}\right)\right| \geq \frac{2 \delta(\varepsilon)}{\varepsilon^{2}} \mathbf{E}\left\|F_{J, f}\right\|_{B},\left\|F_{J, f}\right\|_{B} \leq \frac{2}{\varepsilon^{2}} \mathbf{E}\left\|F_{J, f}\right\|_{B}\right\} d \mu \\
& +\quad \mathbf{P}\left\{\left\|F_{J, f}\right\|_{B}>\frac{2}{\varepsilon^{2}} \mathbf{E}\left\|F_{J, f}\right\|_{B}\right\} \\
& \leq \int_{X} \mathbf{P}\left\{\sup _{n \geq 1}\left|S_{n}\left(F_{J, f}\right)\right| \geq \delta(\varepsilon)\left\|F_{J, f}\right\|_{B}\right\} d \mu+\varepsilon^{2} / 2 \leq \varepsilon^{2} / 2+\varepsilon^{2} / 2=\varepsilon^{2} .
\end{aligned}
$$

Hence,

or

$$
\mu\left\{x \in X: \mathbf{P}\left\{\omega: \sup _{n \geq 1}\left|S_{n}\left(F_{J, f}(\omega, \cdot)\right)(x)\right| \geq \frac{2 \delta(\varepsilon)}{\varepsilon^{2}} \mathbf{E}\left\|F_{J, f}\right\|_{B}\right\} \geq \varepsilon\right\} \leq \varepsilon
$$

$$
\mu\left\{x \in X: \mathbf{P}\left\{\omega: \sup _{n \geq 1}\left|S_{n}\left(F_{J, f}(\omega, \cdot)\right)(x)\right| \leq \frac{2 \delta(\varepsilon)}{\varepsilon^{2}} \mathbf{E}\left\|F_{J, f}\right\|_{B}\right\} \geq 1-\varepsilon\right\} \geq 1-\varepsilon .
$$

By letting $C(\varepsilon)=\frac{2 \delta(\varepsilon)}{\varepsilon^{2}}$, we easily complete the proof.

4.5. Some Gaussian tools. The next lemma is well known in the theory of Gaussian processes. We refer, for instance, to [32, Chap. 10].

Lemma 4.9. Let $X=\left\{X_{t}, t \in T\right\}$ and $Y=\left\{Y_{t}, t \in T\right\}$ be two centered Gaussian processes defined on a finite set $T$.

(a) (Slepian's Lemma). Assume that

$$
\left\|X_{s}-X_{t}\right\|_{2} \leq\left\|Y_{s}-Y_{t}\right\|_{2}
$$

for any $s, t \in T$.

Then

$$
\mathbf{E} f\left[\sup _{T \times T}\left(X_{s}-X_{t}\right)\right] \leq \mathbf{E} f\left[\sup _{T \times T}\left(Y_{s}-Y_{t}\right)\right]
$$

for any positive increasing convex function $f$ on $\mathbb{R}^{+}$.

In particular,

$$
\mathbf{E} \sup _{t \in T} X_{t} \leq \mathbf{E} \sup _{t \in T} Y_{t}
$$

(b) (Sudakov's minoration). There exists a universal constant $B$ such that

$$
\mathbf{E} \sup _{t \in T} X_{t} \geq B \inf _{\substack{s, t \in T \\ s \neq t}}\left\|X_{s}-X_{t}\right\|_{2, \mathbf{P}} \sqrt{\log \#(T)}
$$

for any Gaussian process $X=\left\{X_{t}, t \in T\right\}$. 
(c) (Lower bound for Gaussian norms). Let $X$ be a Gaussian vector and let $N$ be a nonnegative semi-norm. Then

$$
\mathbf{P}\{N(X)<\infty\}=1 \quad \Rightarrow \quad \mathbf{P}\left\{N(X) \geq \frac{1}{2} \mathbf{E} N(X)\right\} \geq c,
$$

where $0<c<1$ is a universal constant.

(d) (Mill's ratio). The Mill's ratio $R(x)=e^{x^{2} / 2} \int_{x}^{\infty} e^{-t^{2} / 2} d t$ verifies

$$
\frac{2}{\sqrt{x^{2}+4}+x} \leq R(x) \leq \frac{2}{\sqrt{x^{2}+\frac{8}{\pi}}+x} \leq \sqrt{\frac{\pi}{2}}
$$

for any $x \geq 0$. It follows that

$$
\mathbf{E} g^{2} \chi\{|g| \geq T\} \leq 6 e^{-T^{2} / 4}
$$

for any standard Gaussian random variable $g$ and any $T>0$.

\section{Proofs}

As clarified in Remark 4.5, we use the random elements $F_{J, f}$ introduced in (4.4) differently, according to the cases $2 \leq p \leq \infty$, in which they are Gaussian, and $1<p<2$, where we choose them $p$-stable.

5.1. Proof of Theorem 3.7. Let $0<\varepsilon<1 / 2$. Let $f \in \mathcal{R}(B)$. By Lemma 4.8, there exists a nonincreasing function $C:] 0,1] \rightarrow \mathbb{R}_{+}$and a set $X_{\varepsilon, J, f}$ of measure greater than $1-\varepsilon$ such that

$$
\mathbf{P}\left\{\omega: \sup _{n \geq 1} \mid S_{n}\left(F_{J, f}(\omega, \cdot)(x) \mid \leq C(\varepsilon) \mathbf{E}\left\|F_{J, f}\right\|_{B}\right\} \geq 1-\varepsilon\right.
$$

for all $x \in X_{\varepsilon, J, f}$. Estimate (3.3) implies that

$$
\mathbf{E} \sup _{n \geq 1}\left|S_{n}\left(F_{J, f}(\omega, \cdot)\right)(x)\right| \leq \frac{4 C(\varepsilon)}{1-\varepsilon} \mathbf{E}\left\|F_{J, f}\right\|_{B} \quad \text { for any } x \in X_{\varepsilon, J, f} .
$$

Recall that $B \subset L^{2}(\mu)$. Let $I$ be a finite set of integers such that $\left\|S_{n}(f)-S_{m}(f)\right\|_{2} \neq 0$ for all $m, n \in I, m \neq n$. By Lemma 4.6 (ii), taking $\mathcal{J}_{0}=\mathbb{N}$, there exists a sub-index $\mathcal{J}$ such that if

$$
A(I)=\left\{\text { for any } J \in \mathcal{J} \text { and for any } n, m \in I, m \neq n, \frac{\left\|S_{n}\left(F_{J, f}\right)-S_{m}\left(F_{J, f}\right)\right\|_{2, \mathbf{P}}}{\left\|S_{n}(f)-S_{m}(f)\right\|_{2, \mu}} \geq \sqrt{1-\varepsilon}\right\},
$$

then $\mu\{A(I)\} \geq \sqrt{1-\varepsilon}$.

By integrating on $X_{\varepsilon, J, f} \cap A(I)$, next using the fact that

$$
\mathbf{E} \sup _{n \in I} S_{n}\left(F_{J, f}\right) \geq 0,
$$

and applying Lemma 4.9(a), we get the estimates

$$
\begin{aligned}
\int_{X_{\varepsilon, J, f}} \mathbf{E} \sup _{n \in I} S_{n}\left(F_{J, f}\right) d \mu & \geq \int_{X_{\varepsilon, J, f} \cap A(I)} \mathbf{E} \sup _{n \in I} S_{n}\left(F_{J, f}\right) d \mu \\
& \geq \sqrt{1-\varepsilon} \mu\left\{X_{\varepsilon, J, f} \cap A(I)\right\} \mathbf{E} \sup _{n \in I} Z\left(S_{n}(f)\right) \\
& \geq \sqrt{1-\varepsilon}(\sqrt{1-\varepsilon}-\varepsilon) \mathbf{E} \sup _{n \in I} Z\left(S_{n}(f)\right) \\
& \geq(1-2 \varepsilon) \mathbf{E} \sup _{n \in I} Z\left(S_{n}(f)\right)
\end{aligned}
$$


for any $J \in \mathcal{J}$. By combining the above estimates, we conclude that

$$
\mathbf{E} \sup _{n \in I} Z\left(S_{n}(f)\right) \leq \frac{1}{(1-2 \varepsilon)} \int_{X_{\varepsilon, J, f}} \mathbf{E} \sup _{n \in I} S_{n}\left(F_{J, f}\right) d \mu \leq K(\varepsilon) \mathbf{E}\left\|F_{J, f}\right\|_{B}
$$

for any $J \in \mathcal{J}$, where

$$
K(\varepsilon)=\frac{4 C(\varepsilon)}{(1-2 \varepsilon)(1-\varepsilon)}
$$

Therefore,

$$
\mathbf{E} \sup _{n \in I} Z\left(S_{n}(f)\right) \leq K(\varepsilon) \inf _{J \in \mathcal{J}} \sup _{H \geq J} \mathbf{E}\left\|F_{H, f}\right\|_{B}=K(\varepsilon) \limsup _{H \rightarrow \infty} \mathbf{E}\left\|F_{H, f}\right\|_{B}
$$

for any $f \in \mathcal{R}(B)$ and finite set $I$. Taking $I=[1, N]$ and letting next $N$ tend to infinity, we get the estimate

$$
\mathbf{E} \sup _{n \geq 1} Z\left(S_{n}(f)\right) \leq K(\varepsilon) \limsup _{H \rightarrow \infty} \mathbf{E}\left\|F_{H, f}\right\|_{B}
$$

5.2. Proof of Theorem 3.4. Let $f \in L^{\infty}(\mu)$. Fubini's theorem and Lemma 4.4 allow us to write

$$
\mathbf{E} \int\left|F_{J, f}\right|^{p} d \mu \leq C_{p}^{p} \int\left(\mathbf{E}\left|F_{J, f}\right|^{2}\right)^{p / 2} d \mu=C_{p}^{p} \int\left(\frac{1}{J} \sum_{j \leq J} T_{j} f^{2}(x)\right)^{p / 2} d \mu(x) .
$$

By the assumption,

$$
\lim _{J \rightarrow \infty}\left\|\frac{1}{J} \sum_{j \leq J} T_{j} f^{2}-\right\| f\left\|_{2, \mu}^{2}\right\|_{1, \mu}=0
$$

By proceeding by extraction, this convergence also holds almost surely along some subsequence $\mathcal{J}_{0}$. Since $\frac{1}{J} \sum_{j \leq J} T_{j} f^{2}(x) \leq\|f\|_{\infty}^{2}$, we further deduce from the dominated convergence theorem that

$$
\lim _{\mathcal{J}_{0} \ni J \rightarrow \infty} \mathbf{E} \int\left(\frac{1}{J} \sum_{j \leq J} T_{j} f^{2}(x)\right)^{p / 2} d \mu=\|f\|_{2, \mu}^{p} .
$$

Let $0<\varepsilon<1$. Extracting, if necessary, from $\mathcal{J}_{0}$ a sub-index which we call again $\mathcal{J}_{0}$, we thus conclude that

$$
\mathbf{E}\left\|F_{J, f}\right\|_{p, \mu} \leq(1+\varepsilon) C_{p}\|f\|_{2, \mu} \quad \text { for any } J \in \mathcal{J}_{0} .
$$

Next the proof is exactly the same as before except that we replace everywhere the norm $\|\cdot\|_{B}$ by the norm $\|\cdot\|_{p, \mu}$. Let $I$ be a finite set of integers. By Lemma 4.6, we can extract from $\mathcal{J}_{0}$ a partial index $\mathcal{J}$ such that an analog of (5.1) holds, namely,

$$
\mathbf{E} \sup _{n \in I} Z\left(S_{n}(f)\right) \leq K(\varepsilon) \mathbf{E}\left\|F_{J, f}\right\|_{p, \mu} \leq C_{p} K(\varepsilon)(1+\varepsilon)\|f\|_{p, \mu}
$$

for any $J \in \mathcal{J}$. A simple approximation argument allows us to get the same inequality for all $f \in L^{p}$. Sudakov's minoration implies that

$$
\sup _{\varrho>0} \varrho \sqrt{\log N_{f}(\varrho)} \leq C_{p} K(\varepsilon)(1+\varepsilon)\|f\|_{p} .
$$


5.3. Proof of Theorem 3.6. (i) By Lemma 4.6 (b), given any index $\mathcal{J}_{0}$, there exists an index $\mathcal{J} \subseteq \mathcal{J}_{0}$ such that

$$
(1-\varepsilon) \mathbf{E} \sup _{n \in I} Z\left(S_{n}(f)\right) \leq \mathbf{E} \int \sup _{n \in I} S_{n}\left(F_{J, f}\right) d \mu
$$

for any $J \in \mathcal{J}$. Moreover,

$$
\mathbf{E} G\left(\sqrt{1-\varepsilon} \sup _{n, m \in I} Z\left(S_{n}(f)\right)-Z\left(S_{m}(f)\right)\right) \leq \mathbf{E} \int_{X} G\left(\sup _{n, m \in I}\left(S_{n}-S_{m}\right)\left(F_{J, f}\right)\right) d \mu
$$

for any positive increasing convex function $G$ on $\mathbb{R}^{+}$and $J \in \mathcal{J}$.

In the following calculation, we put

$$
L=\sup _{\|g\|_{B} \leq 1} \int \sup _{n \in I}\left|S_{n}(g)\right| d \mu
$$

and we let $u_{0}=0$ and $u_{n}=\varepsilon(1+\varepsilon)^{n-1} n \geq 1$. Then

$$
\begin{aligned}
\mathbf{E} \int \sup _{n \in I}\left|S_{n}\left(F_{J, f}\right)\right| d \mu & =\sum_{k=1}^{\infty} \mathbf{E}\left(\mathbf{1}_{u_{k-1} \leq\left\|F_{J, f}\right\|_{B}<u_{k}} \cdot \int \sup _{n \in I}\left|S_{n}\left(F_{J, f}\right)\right| d \mu\right) \\
& \leq \sum_{k=1}^{\infty} \mathbf{P}\left\{u_{k-1} \leq\left\|F_{J, f}\right\|_{B}<u_{k}\right\} \sup _{u_{k-1} \leq\|g\|_{B}<u_{k}} \int \sup _{n \in I}\left|S_{n}(g)\right| d \mu \\
& \leq L \sum_{k=1}^{\infty} u_{k} \mathbf{P}\left\{u_{k-1} \leq\left\|F_{J, f}\right\|_{B}<u_{k}\right\} \\
& \leq L\left(u_{1} \mathbf{P}\left\{\left\|F_{J, f}\right\|_{B}<u_{1}\right\}+(1+\varepsilon) \mathbf{E}\left\|F_{J, f}\right\|_{B} \cdot \mathbf{1}_{u_{1} \leq\left\|F_{J, f}\right\|_{B}}\right) \\
& \leq L\left(\varepsilon+(1+\varepsilon) \mathbf{E}\left\|F_{J, f}\right\|_{B}\right) .
\end{aligned}
$$

By combining and letting next $\varepsilon$ tend to 0 , we get the inequality

$$
\mathbf{E} \sup _{n \in I} Z\left(S_{n}(f)\right) \leq \mathbf{E}\left\|F_{J, f}\right\|_{B} \sup _{\|g\|_{B} \leq 1} \int \sup _{n \in I}\left|S_{n}(g)\right| d \mu
$$

for any $f \in L^{2}(\mu)$. Similarly,

$$
\begin{aligned}
& \mathbf{E} G\left(\sqrt{1-\varepsilon} \sup _{n, m \in I} \mid Z\left(S_{n}(f)\right)\right.\left.-Z\left(S_{m}(f)\right) \mid\right) \\
& \leq \mathbf{E}\left\|F_{J, f}\right\|_{B} \sup _{\|g\|_{B} \leq 1} \mathbf{E} \int_{X} G\left(\sup _{n, m \in I}\left|\left(S_{n}-S_{m}\right)(g)\right|\right) d \mu .
\end{aligned}
$$

(ii) Let $B=L^{p}(\mu)$. We have seen that there exists an index $\mathcal{J}_{0}$ such that

$$
\mathbf{E}\left\|F_{J, f}\right\|_{p, \mu} \leq(1+\varepsilon) C_{p}\|f\|_{2, \mu} \quad \text { for any } J \in \mathcal{J}_{0} .
$$

Therefore,

Moreover,

$$
\sup _{\|f\|_{2, \mu} \leq 1} \mathbf{E} \sup _{n \in I} Z\left(S_{n}(f)\right) \leq C_{p} \sup _{\|g\|_{p, \mu} \leq 1} \int \sup _{n \in I}\left|S_{n}(g)\right| d \mu .
$$

$$
\begin{aligned}
& \sup _{\|f\|_{2, \mu} \leq 1} \mathbf{E} G\left(\sqrt{1-\varepsilon} \sup _{n, m \in I}\left|Z\left(S_{n}(f)\right)-Z\left(S_{m}(f)\right)\right|\right) \\
& \leq C_{p} \sup _{\|g\|_{p, \mu} \leq 1} \mathbf{E} \int_{X} G\left(\sup _{n, m \in I}\left|\left(S_{n}-S_{m}\right)(g)\right|\right) d \mu .
\end{aligned}
$$


5.4. Proof of Theorem 3.12. Let $f \in L^{p}(\mu)$. Let $J$ be any positive integer and let $x \in X$. By (4.5),

$$
\frac{1}{J^{\frac{1}{p}}} \sum_{j \leq J} \theta_{j} T_{j} f(x) \stackrel{\mathcal{D}}{=} \theta_{1}\left(\frac{1}{J} \sum_{j \leq J}\left|T_{j} f(x)\right|^{p}\right)^{1 / p}
$$

Thus,

$$
\mathbf{E}\left|F_{J, f}(x)\right|^{r}=\left(\mathbf{E}\left|\theta_{1}\right|^{r}\right)\left(\frac{1}{J} \sum_{j \leq J}\left|T_{j} f(x)\right|^{p}\right)^{r / p}
$$

for any $r<p$. By Corollary $4.3,\left|T_{j} f(x)\right|^{p} \stackrel{\text { a.e. }}{=} T_{j}|f|^{p}(x)$, so that

$$
\mathbf{E}\left|F_{J, f}(x)\right|^{r}=\left(\mathbf{E}\left|\theta_{1}\right|^{r}\right)\left(\frac{1}{J} \sum_{j \leq J} T_{j}|f|^{p}(x)\right)^{r / p}
$$

for almost all $x$ and all $J \geq 1$. Since trivially $T_{j}|f|^{p} \in L^{1}(\mu)$, we deduce that

$$
\mathbf{E} \int_{X}\left|F_{J, f}(x)\right|^{r} d \mu(x)=\left(\mathbf{E}\left|\theta_{1}\right|^{r}\right) \int_{X}\left(\frac{1}{J} \sum_{j \leq J} T_{j}|f|^{p}(x)\right)^{\frac{r}{p}} d \mu(x) .
$$

Hence,

$$
\begin{aligned}
\left.\left|\mathbf{E} \int_{X}\right| F_{J, f}(x)\right|^{r} d \mu & (x)-\left(\mathbf{E}\left|\theta_{1}\right|^{r}\right)\|f\|_{p, \mu}^{r} \mid \\
& =\left(\mathbf{E}\left|\theta_{1}\right|^{r}\right)\left|\int_{X}\left(\frac{1}{J} \sum_{j \leq J} T_{j}|f|^{p}(x)\right)^{\frac{r}{p}} d \mu(x)-\left(\|f\|_{p, \mu}^{p}\right)^{\frac{r}{p}}\right| \\
& \leq\left(\mathbf{E}\left|\theta_{1}\right|^{r}\right) \int_{X}\left|\left(\frac{1}{J} \sum_{j \leq J} T_{j}|f|^{p}(x)\right)^{\frac{r}{p}}-\left(\|f\|_{p, \mu}^{p}\right)^{\frac{r}{p}}\right| d \mu(x) \\
& \leq\left.\left(\mathbf{E}\left|\theta_{1}\right|^{r}\right) \int_{X}\left|\frac{1}{J} \sum_{j \leq J} T_{j}\right| f\right|^{p}(x)-\left.\|f\|_{p, \mu}^{p}\right|^{\frac{r}{p}} d \mu(x) \\
& \leq\left(\mathbf{E}\left|\theta_{1}\right|^{r}\right)\left(\left.\int_{X}\left|\frac{1}{J} \sum_{j \leq J} T_{j}\right| f\right|^{p}(x)-\|f\|_{p, \mu}^{p} \mid d \mu(x)\right)^{r / p} \rightarrow 0
\end{aligned}
$$

as $J$ tends to infinity by assumption (C). Therefore,

$$
\lim _{J \rightarrow \infty} \mathbf{E} \int_{X}\left|F_{J, f}(x)\right|^{r} d \mu(x)=\left(\mathbf{E}\left|\theta_{1}\right|^{r}\right)\|f\|_{p, \mu}^{r} \quad \text { for any } 0<r<p .
$$

By using Hölder's inequality, we deduce that

$$
\mathbf{E}\left\|F_{J, f}\right\|_{r, \mu} \leq\left(\mathbf{E} \int\left|F_{J, f}(x)\right|^{r} d \mu\right)^{1 / r} \leq 2\left\|\theta_{1}\right\|_{r}\|f\|_{p, \mu}
$$

for all $J \geq J_{0}$, say.

By assumption, property $\left(\mathcal{B}_{r}\right)$ holds for some $1<r<p$. From Lemma 4.8 it follows that there exists a nonincreasing function $C:] 0,1] \rightarrow \mathbb{R}_{+}$such that for any $f \in L^{r}(\mu)$, any $J \geq 1$, and any $0<\varepsilon<1$, there exists a measurable set $\widetilde{X}=\widetilde{X}_{\varepsilon, J, f}$ of measure greater than $1-\sqrt{\varepsilon}$ such that

$$
\mathbf{P}\left\{\omega: \sup _{n \geq 1}\left|S_{n}\left(F_{J, f}(\omega, \cdot)\right)(x)\right|>C(\varepsilon)\left\|F_{J, f}\right\|_{r, \mu}\right\} \leq \varepsilon
$$


for all $x \in \tilde{X}$. In what follows, we assume that $0<\varepsilon<1 / 6$. Let $\delta(\varepsilon)=C(\varepsilon) / \varepsilon$. Let also $x \in \widetilde{X}$ and $J \geq J_{0}$. Using Chebyshev's inequality and (5.4), we get the estimates

$$
\begin{gathered}
\mathbf{P}\left\{\omega: \sup _{n \geq 1}\left|S_{n}\left(F_{J, f}(\omega, \cdot)\right)(x)\right|>2 \delta(\varepsilon)\left\|\theta_{1}\right\|_{r}\|f\|_{p, \mu}\right\} \\
\leq \mathbf{P}\left\{\omega: \sup _{n \geq 1}\left|S_{n}\left(F_{J, f}(\omega, \cdot)\right)(x)\right|>\delta(\varepsilon) \mathbf{E}\left\|F_{J, f}\right\|_{r, \mu}\right\} \\
\leq \mathbf{P}\left\{\left\|F_{J, f}\right\|_{r, \mu}>\mathbf{E}\left\|F_{J, f}\right\|_{r, \mu} / \varepsilon\right\}+\mathbf{P}\left\{\omega: \sup _{n \geq 1}\left|S_{n}\left(F_{J, f}(\omega, \cdot)\right)(x)\right|>\delta(\varepsilon) \mathbf{E}\left\|F_{J, f}\right\|_{r, \mu},\left\|F_{J, f}\right\|_{r, \mu}\right. \\
\left.\leq \mathbf{E}\left\|F_{J, f}\right\|_{r, \mu} / \varepsilon\right\} \\
\leq \varepsilon+\mathbf{P}\left\{\omega: \sup _{n \geq 1}\left|S_{n}\left(F_{J, f}(\omega, \cdot)\right)(x)\right|>C(\varepsilon)\left\|F_{J, f}\right\|_{r, \mu}\right\} \leq 2 \varepsilon .
\end{gathered}
$$

Therefore,

$$
\begin{array}{r}
\mathbf{P}\left\{\omega: \sup _{n \geq 1}\left|S_{n}\left(F_{J, f}(\omega, \cdot)\right)(x)\right| \leq 2 \delta(\varepsilon)\left\|\theta_{1}\right\|_{r}\|f\|_{p, \mu}\right\} \geq 1-2 \varepsilon, \\
\text { for any } x \in \widetilde{X} \quad \text { and any } J \geq J_{0}
\end{array}
$$

Let $\delta$ be some fixed positive real. Let $I$ be a finite set of positive integers and let $M=\#\{I\}$. Assume that $\left\|S_{n}(f)-S_{m}(f)\right\|_{p, \mu} \geq \delta$ if $n \neq m, n, m \in I$. By Lemma 4.6 (i), there exists an index $\mathcal{J}$ and a measurable set $A=A_{\varepsilon, I, f}$ such that $\mu\{A\} \geq 1-\varepsilon$, and further, for all $x \in A$, the following inequalities:

$$
\begin{aligned}
(1-\varepsilon)^{1 / p}\left\|\theta_{1}\right\|_{r}\left\|S_{n}(f)-S_{m}(f)\right\|_{p, \mu} & \leq\left\|\left(S_{n}-S_{m}\right)\left(F_{J, f}\right)(x)\right\|_{r, \mathbf{P}} \\
& \leq(1+\varepsilon)^{1 / p}\left\|\theta_{1}\right\|_{r}\left\|S_{n}(f)-S_{m}(f)\right\|_{p, \mu}
\end{aligned}
$$

are satisfied for all $J \in \mathcal{J}$, all $n, m \in I$, and all $r<p$. Set

$$
Y=Y_{\varepsilon, I, J, f}=\tilde{X} \cap A \text {. }
$$

For each $x$ fixed, the process

$$
S_{J, f, x}(\omega, n)=\frac{1}{J^{1 / p}} \sum_{j \leq J} \theta_{j}(\omega) T_{j} S_{n}(f)(x), \quad n \geq 1,
$$

is a $p$-stable random function. Further, the process

$$
\mathcal{S}_{J, f, x}\left(\omega^{\prime}, \omega^{\prime \prime}, n\right)=\frac{1}{J^{1 / p}} \sum_{1 \leq j \leq J} \eta_{j}\left(\omega^{\prime}\right) g_{j}\left(\omega^{\prime \prime}\right) T_{j} S_{n} f(x), \quad n \geq 1,
$$

has the same distribution as $\left\{S_{J, f, x}(\cdot, n), n \geq 1\right\}$. Recall (see Sec. 4.2) that we have underlying joint probability spaces $\left(\Omega^{\prime}, \mathcal{B}^{\prime}, \mathbf{P}^{\prime}\right)$ and $\left(\Omega^{\prime \prime}, \mathcal{B}^{\prime \prime}, \mathbf{P}^{\prime \prime}\right)$ on which the sequence $\left\{\eta_{j}, j \geq 1\right\}$ and the sequence $\left\{g_{j}, j \geq 1\right\}$ of i.i.d. Gaussian standard random variables are respectively defined. Here we take both sequences infinite.

Thus, (5.6) reads as follows:

$$
\mathbf{P}^{\prime} \times \mathbf{P}^{\prime \prime}\left\{\left(\omega^{\prime}, \omega^{\prime \prime}\right): \sup _{n \geq 1}\left|\mathcal{S}_{J, f, x}\left(\omega^{\prime}, \omega^{\prime \prime}, n\right)\right| \leq 2 \delta(\varepsilon)\left\|\theta_{1}\right\|_{r}\|f\|_{p, \mu}\right\} \geq 1-2 \varepsilon
$$

for all $x \in \widetilde{X}$, and all $J \geq J_{0}$. Let

$$
H\left(\omega^{\prime}\right)=\mathbf{P}^{\prime \prime}\left\{\omega^{\prime \prime}: \sup _{n \geq 1}\left|\mathcal{S}_{J, f, x}\left(\omega^{\prime}, \omega^{\prime \prime}, n\right)\right| \leq 2 \delta(\varepsilon)\left\|\theta_{1}\right\|_{r}\|f\|_{p, \mu}\right\} .
$$


By Fubini's theorem, the left-hand term in (5.7) also writes as

$$
\begin{aligned}
\int_{\Omega^{\prime}} H\left(\omega^{\prime}\right) d \mathbf{P}^{\prime}\left(\omega^{\prime}\right) & =\int_{\omega^{\prime}: H\left(\omega^{\prime}\right) \leq \varepsilon} H\left(\omega^{\prime}\right) d \mathbf{P}^{\prime}\left(\omega^{\prime}\right)+\int_{\omega^{\prime}: H\left(\omega^{\prime}\right)>\varepsilon} H\left(\omega^{\prime}\right) d \mathbf{P}^{\prime}\left(\omega^{\prime}\right) \\
& \leq \varepsilon+\mathbf{P}^{\prime}\left\{\omega^{\prime}: H\left(\omega^{\prime}\right)>\varepsilon\right\} .
\end{aligned}
$$

Hence,

$$
\mathbf{P}^{\prime}\left\{\omega^{\prime}: \mathbf{P}^{\prime \prime}\left\{\omega^{\prime \prime}: \sup _{n \geq 1}\left|\mathcal{S}_{J, f, x}\left(\omega^{\prime}, \omega^{\prime \prime}, n\right)\right| \leq 2 \delta(\varepsilon)\left\|\theta_{1}\right\|_{r}\|f\|_{p, \mu}\right\} \geq \varepsilon\right\} \geq 1-3 \varepsilon .
$$

For each fixed $\omega^{\prime} \in \Omega^{\prime},\left\{\mathcal{S}_{J, f, x}\left(\omega^{\prime}, \cdot, n\right), n \geq 1\right\}$ is a Gaussian process. Let $\mathbf{E}_{\mathbf{P}^{\prime \prime}}$ denote the expectation symbol with respect to $\mathbf{P}^{\prime \prime}$. Estimate (3.3) implies that

$$
\left.1-3 \varepsilon \leq \mathbf{P}^{\prime}\left\{\omega^{\prime}: \mathbf{E}_{\mathbf{P}^{\prime \prime}} \sup _{n \geq 1} \mid \mathcal{S}_{J, f, x}\left(\cdot, \omega^{\prime}, x\right)\right) \mid \leq \frac{8 \delta(\varepsilon)}{\varepsilon}\left\|\theta_{1}\right\|_{r}\|f\|_{p, \mu}\right\}
$$

for every $x \in X_{\varepsilon, J, f}$. Write for a while

$$
\begin{aligned}
D(\omega, n, m) & =D_{J, f, x}(\omega, n, m)=S_{J, f, x}(\omega, n)-S_{J, f, x}(\omega, m), \\
\mathcal{D}\left(\omega^{\prime}, \omega^{\prime \prime}, n, m\right) & =\mathcal{D}_{J, f, x}\left(\omega^{\prime}, \omega^{\prime \prime}, n, m\right)=\mathcal{S}_{J, f, x}\left(\omega^{\prime}, \omega^{\prime \prime}, n\right)-\mathcal{S}_{J, f, x}\left(\omega^{\prime}, \omega^{\prime \prime}, m\right),
\end{aligned}
$$

and

$$
\Delta(n, m)=\Delta_{J, f, x}(n, m)=\left(\frac{1}{J} \sum_{1 \leq j \leq J}\left|T_{j}\left(S_{n}-S_{m}\right) f(x)\right|^{p}\right)^{1 / p}
$$

By (4.3),

$$
\mathbf{E}_{\mathbf{P}^{\prime}} \mathbf{E}_{\mathbf{P}^{\prime \prime}} e^{i t \mathcal{D}\left(\omega^{\prime}, \omega^{\prime \prime}, n, m\right)}=\mathbf{E}_{\mathbf{P}} e^{i t\left(S_{J, f, x}(., n)-S_{J, f, x}(., m)\right)}=\mathbf{E}_{\mathbf{P}} e^{i t \theta_{1} \Delta(n, m)}=e^{-|t|^{p} \Delta(n, m)^{p}} .
$$

Since $\mathbf{E} e^{i t g}=e^{-t^{2} \tau^{2} / 2}$, where $\tau=\left(\mathbf{E} g^{2}\right)^{1 / 2}$, we get from (4.2) the equalities

$$
\mathbf{E}_{\mathbf{P}^{\prime}} \mathbf{E}_{\mathbf{P}^{\prime \prime}} e^{i t \mathcal{D}\left(\omega^{\prime}, \omega^{\prime \prime}, n, m\right)}=\mathbf{E}_{\mathbf{P}^{\prime}} e^{-t^{2}\left\|\mathcal{D}\left(\omega^{\prime}, \cdot, n, m\right)\right\|_{2, \mathbf{P}^{\prime \prime}}^{2} / 2}=e^{-|t|^{p} \Delta(n, m)^{p}}
$$

Put for each $\omega^{\prime} \in \Omega^{\prime}$

$$
d_{J, \omega^{\prime}, x}(n, m)=\left\|\mathcal{D}_{J, f, x}\left(\omega^{\prime}, \cdot, n, m\right)\right\|_{2, \mathbf{P}^{\prime \prime}}
$$

Moreover, let

$$
d_{J, x}(n, m)=\left(\frac{1}{J} \sum_{j \leq J} T_{j}\left|S_{n}(f)-S_{m}(f)\right|^{p}(x)\right)^{1 / p} .
$$

We note that $d_{J, x}(n, m)=\Delta_{J, f, x}(n, m)$ for almost all $x \in X$. Further,

$$
\mathbf{E}_{\mathbf{P}^{\prime}} e^{-t^{2} d_{J, \omega^{\prime}, x}(n, m)^{2} / 2}=e^{-|t|^{p} d_{J, x}(n, m)^{p}} \text {. }
$$

Then

$$
\begin{aligned}
& \mathbf{P}\left\{\text { there exist } n, m \in I: d_{J, \omega^{\prime}, x}(n, m)<\varepsilon d_{J, x}(n, m)\right\} \\
& \quad \leq \sum_{n, m \in I} \mathbf{P}\left\{e^{-t^{2} d_{J, \omega^{\prime}, x}(n, m) / 2}>e^{-t^{2} \varepsilon^{2} d_{J, x}^{2}(n, m)}\right\} \\
& \leq M^{2} e^{t^{2} \varepsilon^{2} d_{J, x}^{2}(n, m)-|t|^{p} d_{J, x}(n, m)^{p}}
\end{aligned}
$$

and so,

$$
\mathbf{P}\left\{\text { there exist } n, m \in I: d_{J, \omega^{\prime}, x}(n, m)<\varepsilon d_{J, x}(n, m)\right\} \leq M^{2} \inf _{t>0} e^{t^{2} \varepsilon^{2} d_{J, x}^{2}(n, m)-|t|^{p} d_{J, x}(n, m)^{p}} .
$$


The function $\varphi(t)=e^{t^{2} a-t^{p} b}$ has an extremum at the value $t^{*}=\left(\frac{p b}{2 a}\right)^{\frac{1}{2-p}}$, and

$$
\varphi\left(t^{*}\right)=\exp \left\{a^{-\frac{p}{2-p}} b^{\frac{2}{2-p}}\left[(p / 2)^{\frac{2}{2-p}}-(p / 2)^{\frac{p}{2-p}}\right]\right\} .
$$

Applying this with $a=\varepsilon^{2} d_{J, x}^{2}(n, m)$ and $b=d_{J, x}(n, m)^{p}$, we get the estimates

$$
\begin{aligned}
& \mathbf{P}\left\{\text { there exist } n, m \in I: d_{J, \omega^{\prime}, x}(n, m)<\varepsilon d_{J, x}(n, m)\right\} \\
& \leq M^{2} \exp \left\{\varepsilon^{-\frac{2 p}{2-p}}\left(d_{J, x}(n, m)\right)^{-\frac{2 p}{2-p}} d_{J, x}(n, m)^{\frac{2 p}{2-p}}\left[(p / 2)^{\frac{2}{2-p}}-(p / 2)^{\frac{p}{2-p}}\right]\right\} \\
& :=M^{2} \exp \left\{-\varepsilon^{-\frac{2 p}{2-p}} C(p)\right\}
\end{aligned}
$$

with $C(p)=(p / 2)^{\frac{p}{2-p}}-(p / 2)^{-\frac{2}{2-p}}>0$. Choose $\varepsilon=(\tau \log M)^{-\frac{2-p}{2 p}}$. We get the estimates

$$
\mathbf{P}\left\{\text { there exist } n, m \in I: d_{J, \omega^{\prime}, x}(n, m)<(\tau \log M)^{-\frac{2-p}{2 p}} d_{J, x}(n, m)\right\} \leq M^{2-\tau C(p)} \leq \frac{1}{2}
$$

for $\tau=\tau(p)$ depending on $p$ only and small enough.

Now, if $x \in Y$, then

$$
\left\|\left(S_{n}-S_{m}\right)\left(F_{J, f}\right)(x)\right\|_{r, \mathbf{P}^{\prime} \times \mathbf{P}^{\prime \prime}} \geq c(\varepsilon, r, p)\left\|S_{n}(f)-S_{m}(f)\right\|_{p, \mu}
$$

for all $J \in \mathcal{J}$, all $n, m \in I, m \neq n$, and all $r<p$. Since

$$
\begin{gathered}
\left(S_{n}-S_{m}\right)\left(F_{J, f}\right)(x) \stackrel{\mathcal{D}}{=}\left(\mathcal{S}_{J, f, x}(n)-\left(\mathcal{S}_{J, f, x}(m)\right),\right. \\
\left\|\left(S_{n}-S_{m}\right)\left(F_{J, f}\right)(x)\right\|_{r, \mathbf{P}^{\prime} \times \mathbf{P}^{\prime \prime}}=\left\|\theta_{1}\right\|_{r} d_{J, x}(n, m),
\end{gathered}
$$

whence

$$
d_{J, x}(n, m) \geq c(\varepsilon, r, p)\left\|S_{n}(f)-S_{m}(f)\right\|_{p, \mu}
$$

for all $J \in \mathcal{J}$ and all $n, m \in I, m \neq n$.

Putting together (5.10) and (5.9) implies that there exists a measurable set $\Omega_{0}^{\prime}$ with $\mathbf{P}^{\prime}\left(\Omega_{0}^{\prime}\right)>0$ such that

$$
d_{J, \omega^{\prime}, x}(n, m) \geq c(\varepsilon, r, p) \frac{d_{J, x}(n, m)}{(\log \#\{I\})^{1 / p-1 / 2}} \geq c(\varepsilon, r, p) \frac{\delta}{(\log \#\{I\})^{1 / p-1 / 2}}
$$

for any $\omega^{\prime} \in \Omega_{0}^{\prime}$, and all $n, m \in I$. By Sudakov's inequality,

$$
\begin{aligned}
\|f\|_{p, \mu} & \left.\geq c(r, p) \mathbf{E}_{\mathbf{P}^{\prime \prime}} \sup _{n \in I} \mid \mathcal{S}_{J, f, x}\left(\cdot, \omega^{\prime}, x\right)\right) \mid \\
& \geq c(r, p) \delta(\log \#\{I\})^{1 / 2+1 / 2-1 / p} .
\end{aligned}
$$

A routine argument together with (5.9) now easily leads to the estimate

$$
\|f\|_{p, \mu} \geq c(r, p) \sup _{\delta>0} \delta\left(\log N_{f}^{p}(\delta)\right)^{1 / q}
$$

where $c(r, p)>0$ depends on $r$ and $p$ only. It is only at this last stage that the fact that $p>1$ is necessary. 
5.5. Proof of Theorem 3.3. Let $f \in L^{\infty}(\mu)$ be such that $\|f\|_{2}=1$. Let $I$ be a finite subset of $\mathbb{N}$ and let $M=\#\{I\}$. Write for a while $N=N_{\omega}=\left|S_{n}\left(F_{J, f}(\omega, \cdot)\right)\right|$ and $\beta(\omega)=\mu\{x$ : $\left.N_{\omega}(x) \geq \frac{1}{2} \mathbf{E} N_{\omega}(x)\right\}$. By Lemma $4.9(\mathrm{c})$,

$$
\mathbf{P}\left\{N_{\omega}(x) \geq \frac{1}{2} \mathbf{E} N_{\omega}(x)\right\} \geq c
$$

for each $x$. Thus,

We have the estimates

$$
\mu \otimes \mathbf{P}\left\{(\omega, x): N_{\omega}(x) \geq \frac{1}{2} \mathbf{E} N_{\omega}(x)\right\} \geq c .
$$

$$
c \leq \mathbf{E} \beta=\mathbf{E} \beta\left(\chi_{\{\beta \geq c / 2\}}+\chi_{\{\beta \leq c / 2\}}\right) \leq c / 2+\mathbf{P}\{\beta \geq c / 2\} .
$$

Hence, $\mathbf{P}\{\beta \geq c / 2\} \geq c / 2$, and using the previous notation, we deduce that for each $J \geq 1$ there exists a measurable set $D_{J}$ of probability larger than $c / 2$ such that

$$
\mu\left\{x:\left|S_{n}\left(F_{J, f}(\omega, \cdot)\right)(x)\right| \geq \frac{1}{2} \mathbf{E}\left|S_{n}\left(F_{J, f}\right)(x)\right|\right\} \geq c / 2 \text { for any } \omega \in D_{J} .
$$

Let $0<\gamma<1$ be fixed. By Lemma 4.6(ii), there exists an index $\mathcal{J}$ and a measurable set $A$ with $\mu\{A\} \geq \gamma^{2}$ such that for all $x \in A$,

$$
\gamma \mathbf{E} \sup _{n \in I} Z\left(S_{n}(f)\right) \leq \mathbf{E} \int \sup _{n \in I} S_{n}\left(F_{J, f}\right) d \mu \quad \text { for any } J \in \mathcal{J}
$$

Hence,

$$
\mu\left\{x:\left|S_{n}\left(F_{J, f}(\omega, \cdot)\right)(x)\right| \geq \frac{\gamma}{2} \mathbf{E} \sup _{n \in I} Z\left(S_{n}(f)\right)\right\} \geq c / 3 \quad \text { for any } \omega \in D_{J},
$$

assuming that $\gamma$ is sufficiently close to 1 and all $J \in \mathcal{J}$ are greater than some sufficiently large number, which we do.

We simplify the notation in what follows and write $F_{J}=F_{J, f}$. Put for any $A>0$

$$
E_{A}=\left\{(\omega, x) \in \Omega \times X:\left|F_{J}(\omega, x)\right| \leq A\right\} \text { and } E_{A, \omega}=\left\{x \in X:(\omega, x) \in E_{A}\right\}
$$

and let for any $\omega \in \Omega$ and $x \in X$,

$$
F_{A, J}(x)=F_{A, J, \omega}(x)=F_{J}(\omega, x) \cdot \mathbf{1}_{E_{A, \omega}}(x)
$$

and

$$
F^{A, J}(x)=F^{A, J, \omega}(x)=F_{J}(\omega, x) \cdot \mathbf{1}_{E_{A, \omega}^{c}}(x)
$$

Obviously,

$$
\mathbf{E} \int \sup _{n \in I} S_{n}\left(F_{J, f}\right) d \mu \leq \mathbf{E} \int \sup _{n \in I}\left|S_{n}\left(F^{A, J}\right)\right| d \mu+\mathbf{E} \int \sup _{n \in I}\left|S_{n}\left(F_{A, J}\right)\right| d \mu .
$$

By definition, $F_{A, J, \omega}(\cdot)$ (respectively, $\left.F^{A, J, \omega}(\cdot)\right)$ is $\mathcal{A}$-measurable. Since $f \in L^{\infty}(\mu)$,

$$
\mathbf{P}\left\{\omega: F_{A, J, \omega}(\cdot) \text { and } F^{A, J, \omega}(\cdot) \in L^{\infty}(\mu)\right\}=1 .
$$

Since $\max _{i \leq n} x_{i} \leq\left(\sum_{i \leq n} x_{i}^{2}\right)^{1 / 2}$ for any nonnegative real numbers, by using twice CauchySchwarz's inequality and next Fubini's inequality, we get the estimates

$$
\begin{aligned}
\mathbf{E} \int \sup _{n \in I}\left|S_{n}\left(F^{A, J}\right)\right| d \mu & \leq \mathbf{E}\left(\sum_{n \in I} \int\left|S_{n}\left(F^{A, J}\right)\right|^{2} d \mu\right)^{1 / 2} \\
& \leq\left(\sum_{n \in I} \int \mathbf{E}\left|S_{n}\left(F^{A, J}\right)\right|^{2} d \mu\right)^{1 / 2} \leq \sqrt{M} \mathbf{E}\left\|F^{A, J}\right\|_{2, \mu} .
\end{aligned}
$$


We have to estimate $\left\|F^{A, J}\right\|_{2, \mu}$. By Fubini's theorem and then Lemma 4.9(d) applied with $g=F_{J, f} /\left\|F_{J, f}\right\|_{2, \mathbf{P}}$ and $T=A /\left\|F_{J, f}\right\|_{2, \mathbf{P}}$,

$$
\begin{aligned}
\mathbf{E}\left\|F^{A, J}\right\|_{2, \mu}^{2} & =\int_{X} \mathbf{E}\left|F_{J, f}(x)\right|^{2} \cdot \mathbf{1}_{\left(\left|F_{J, f}(x)\right| \geq A\right)} d \mu(x) \\
& \leq 6 \int_{X}\left\|F_{J, f}(x)\right\|_{2, \mathbf{P}}^{2} \exp \left\{-\frac{A^{2}}{4\left\|F_{J, f}(x)\right\|_{2, \mathbf{P}}^{2}}\right\} d \mu(x) .
\end{aligned}
$$

We have $\left\|F_{J, f}(x)\right\|_{2, \mathbf{P}}^{2} \stackrel{\text { a.e. }}{=} \frac{1}{J} \sum_{j \leq J} T_{j}\left(f^{2}\right)(x)$. By assumption $(\mathrm{C}), \frac{1}{J} \sum_{j \leq J} T_{j} f^{2}$ converges to 1 in $L^{1}(\mu)$ along some subsequence extracted from $\mathcal{J}$; we can make this convergence almost everywhere too. The requirement that $f \in L^{\infty}(\mu)$, together with the dominated convergence theorem then implies that

$$
\int_{X}\left\|F_{J, f}(x)\right\|_{2, \mathbf{P}}^{2} \exp \left\{-\frac{A^{2}}{4\left\|F_{J, f}(x)\right\|_{2, \mathbf{P}}^{2}}\right\} d \mu(x) \rightarrow \exp \left\{-A^{2} / 4\right\}
$$

along this index.

Extracting again, if necessary, we conclude that

$$
\mathbf{E}\left\|F^{A, J}\right\|_{2, \mu}^{2} \leq 2 \exp \left\{-A^{2} / 4\right\}
$$

along some index, which we still denote by $\mathcal{J}$. Choose now $A=\sqrt{8 \log M}$. We get the estimates

$$
\mathbf{E} \int \sup _{n \in I}\left|S_{n}\left(F^{A, J, \omega}\right)\right| d \mu \leq 9 \sqrt{M} \exp \left\{-A^{2} / 8\right\} \leq 9 M^{-1 / 2} .
$$

Assume that

$$
\min _{\substack{n, m \in I \\ n \neq m}}\left\|S_{n}(f)-S_{m}(f)\right\|_{2, \mu} \geq \delta .
$$

Using Lemma 4.9(b), we get the inequalities

$$
\mu\left\{x: \sup _{n \in I}\left|S_{n}\left(F_{A, J, \omega}\right)(x)\right| \geq \frac{\gamma B \delta}{2} \sqrt{\log M}-9 M^{-1 / 2}\right\} \geq c / 3 \text { for any } \omega \in D_{J}
$$

for all $J \in \mathcal{J}$. Let

It follows that

$$
\phi_{I, J, \omega}=\frac{F_{A, J, \omega}}{A}
$$

$$
\mu\left\{x: \sup _{n \in I}\left|S_{n}\left(\phi_{I, J, \omega}\right)(x)\right| \geq c^{\prime} \delta\right\} \geq c / 3 \quad \text { for any } \omega \in D_{J}
$$

where $c^{\prime}$ is a positive universal constant. Assume that $C(\delta)=\infty$ for some $\delta>0$. This means that we can select sets $I$ verifying (5.19) with cardinality $M$ as large as we wish. But

$$
\begin{aligned}
d\left(S^{*}\left(\phi_{I, J, \omega}\right), 0\right) & \geq d\left(\sup _{n \in I} \mid S_{n}\left(\phi_{I, J, \omega}\right), 0\right) \\
& \geq \int_{\sup _{n \in I} \mid S_{n}\left(\phi_{I, J, \omega}\right) \geq c^{\prime} \delta} \frac{\sup _{n \in I}\left|S_{n}\left(\phi_{I, J, \omega}\right)\right|}{1+\sup _{n \in I}\left|S_{n}\left(\phi_{I, J, \omega}\right)\right|} d \mu \\
& \geq(c / 3) \frac{c^{\prime} \delta}{1+c^{\prime} \delta} .
\end{aligned}
$$

Moreover,

$$
\mathbf{E}\left\|\phi_{I, J, \omega}\right\|_{2, \mu}^{2} \leq \frac{1}{8 \log M} \mathbf{E} \int\left|F_{j, f}\right|^{2} d \mu \leq \frac{1}{8 \log M} .
$$


Hence, on a subset $D_{J}^{\prime}$ of $D_{J}$ of positive measure, we have the estimates

$$
\left\|\phi_{I, J, \omega}\right\|_{\infty, \mu} \leq 1 \text { and }\left\|\phi_{I, J, \omega}\right\|_{2, \mu} \leq K / \sqrt{\log M} .
$$

Moreover, $K$ depends on $c$ only. Picking $\omega$ in $D_{J}^{\prime}$ and varying $J$, we deduce that $S^{*}$ cannot be continuous at 0 . Hence, we get a contradiction with (3.2). This completes the proof.

5.6. Proof of Theorem 3.10. We start as in the proof of Theorem 3.3. By using exactly the same arguments for proving (5.17), we get here the estimates

$$
\begin{aligned}
\mathbf{E} \int \sup _{n \in I}\left|S_{n}\left(F^{A, J}\right)\right| d \mu & \leq \mathbf{E}\left(\sum_{n \in I} \int\left|S_{n}\left(F^{A, J}\right)\right|^{2} d \mu\right)^{1 / 2} \\
& \leq\left(\sum_{n \in I} \int \mathbf{E}\left|S_{n}\left(F^{A, J}\right)\right|^{2} d \mu\right)^{1 / 2} \leq \sqrt{M} S_{1}(I) \mathbf{E}\left\|F^{A, J}\right\|_{2, \mu} .
\end{aligned}
$$

Next, estimate (5.18) is modified as follows. Let $\alpha>1$ be some fixed real. By extracting, we conclude that $\mathbf{E}\left\|F^{A, J}\right\|_{2, \mu}^{2} \leq \alpha \exp \left\{-A^{2} / 4\right\}$ along some index, still denoted $\mathcal{J}$. Thus, by $(5.23)$,

$$
\mathbf{E} \int \sup _{n \in I}\left|S_{n}\left(F^{A, J, \omega}\right)\right| d \mu \leq \sqrt{M} S_{1}(I) \mathbf{E}\left\|F^{A, J}\right\|_{2, \mu} \leq 6 \sqrt{\alpha M} S_{1}(I) \exp \left\{-A^{2} / 8\right\} .
$$

Let $\left.\delta=\min \left\{(\alpha-1) e^{-A^{2} / 4 \alpha}, 1\right)\right\}$ and $\delta_{k}=\delta 2^{-k}, k \geq 1$. We can extract from $\mathcal{J}$ a subsequence $\mathcal{J}^{*}=\left\{J_{k}, k \geq 1\right\}$ depending on $f$ and $\alpha$ and such that

$$
\mu\left\{\left|\frac{1}{J_{k}} \sum_{j \leq J_{k}} T_{j} f^{2}-1\right|>\delta_{k}\right\} \leq \delta_{k} \quad \text { for all } k \geq 1
$$

Put

$$
B=\left\{\text { for any } k \geq 1,\left|\frac{1}{J_{k}} \sum_{j \leq J_{k}} T_{j} f^{2}-1\right| \leq \delta_{k}\right\}
$$

Plainly,

$$
\mathbf{E} \int \sup _{n \in I}\left|S_{n}\left(F_{A, J}\right)\right| d \mu \leq \mathbf{E} \int_{B} \sup _{n \in I}\left|S_{n}\left(F_{A, J}\right)\right| d \mu+\mathbf{E} \int_{B^{c}} \sup _{n \in I}\left|S_{n}\left(F_{A, J}\right)\right| d \mu .
$$

The first integral in the right-hand side of (5.25) can be bounded for any $R>0$ by

$$
\int_{B} \mathbf{E}\left(\sup _{n \in I}\left|S_{n}\left(F_{A, J}\right)\right| \mathbf{1}_{\left\{\left\|F_{A, J}\right\|_{2, \mu}>R\right\}}\right) d \mu+\int_{B} \mathbf{E}\left(\sup _{n \in I}\left|S_{n}\left(F_{A, J}\right)\right| \mathbf{1}_{\left\{\left\|F_{A, J}\right\|_{2, \mu} \leq R\right\}}\right) d \mu .
$$

Consider the first integral in (5.26). The fact that $S_{n}$ is continuous on $L^{\infty}(\mu)$ and Chebyshev's inequality allow us to write

$$
\begin{gathered}
\int_{B} \mathbf{E}\left(\sup _{n \in I}\left|S_{n}\left(F_{A, J}\right)\right| \mathbf{1}_{\left\{\left\|F_{A, J}\right\|_{2, \mu}>R\right\}}\right) d \mu \leq \mathbf{E}\left(\left\|\sup _{n \in I} \mid S_{n}\left(F_{A, J}\right)\right\|_{\infty, \mu} \cdot \mathbf{1}_{\left\{\left\|F_{A, J}\right\|_{2, \mu}>R\right\}}\right) \\
\leq A S_{2}(I) \mathbf{P}\left\{\left\|F_{A, J}\right\|_{2, \mu}>R\right\} \leq A S_{2}(I) e^{-R^{2} / 4 \alpha} \mathbf{E} \exp \left\{\frac{1}{4 \alpha}\left\|F_{A, J}\right\|_{2, \mu}^{2}\right\} .
\end{gathered}
$$

We claim that

$$
\mathbf{E} \exp \left\{\frac{1}{4 \alpha}\left\|F_{A, J}\right\|_{2, \mu}^{2}\right\} \leq \sqrt{2}+\alpha-1
$$

for any $J \in \mathcal{J}^{*}$. Admit this for a while. Then

$$
\int_{B} \mathbf{E}\left(\sup _{n \in I}\left|S_{n}\left(F_{A, J}\right)\right| \mathbf{1}_{\left\{\left\|F_{A, J}\right\|_{2, \mu}>R\right\}}\right) d \mu \leq A S_{2}(I) e^{-R^{2} / 4 \alpha}(\sqrt{2}+\alpha-1) .
$$


Now we prove (5.28). Let $a=\frac{1}{4 \alpha}$. At first, by using Jensen's inequality,

$$
\begin{aligned}
\mathbf{E} \exp \left\{a\left\|F_{A, J}\right\|_{2, \mu}^{2}\right\} & =\mathbf{E} \exp \left\{a \int_{X} F_{A, J}^{2} d \mu\right\} \leq \mathbf{E} \int_{X} \exp \left\{a F_{A, J}^{2}\right\} d \mu \\
& \leq \mathbf{E} \int_{B} \exp \left\{a F_{A, J}^{2}\right\} d \mu+e^{a A^{2}} \mu\left(B_{\alpha}^{c}\right) .
\end{aligned}
$$

Next, on $B$, we have $\frac{1}{J} \sum_{j \leq J} T_{j} f^{2} \leq 1+\delta<\alpha$, so that

$$
1-2 a\left(\frac{1}{J} \sum_{j \leq J} T_{j} f^{2}\right)>1-2 a \alpha=\frac{1}{2} \quad \text { for all } J \in \mathcal{J}^{*} .
$$

Since $\mathbf{E} e^{b g^{2}}=\frac{1}{\sqrt{1-2 b}}$ if $0 \leq b<\frac{1}{2}$, we get the estimate

$$
\int_{B} \mathbf{E} \exp \left\{a F_{A, J}^{2}\right\} d \mu \leq \int_{B} \mathbf{E} \exp \left\{a F_{J}^{2}\right\} d \mu=\int_{B} \frac{d \mu}{\sqrt{1-2 a\left(\frac{1}{J} \sum_{j \leq J} T_{j} f^{2}\right)}} \leq \sqrt{2} .
$$

Hence,

$$
\mathbf{E} \exp \left\{a\left\|F_{A, J}\right\|_{2, \mu}^{2}\right\} \leq \sqrt{2}+e^{a A^{2}} \mu\left(B^{c}\right) \leq \sqrt{2}+\delta e^{A^{2} a} \leq \sqrt{2}+\alpha-1
$$

for any $J \in \mathcal{J}^{*}$.

For the second integral in (5.26), we have the straightforward bound

$$
\int_{B} \mathbf{E}\left(\sup _{n \in I}\left|S_{n}\left(F_{A, J}\right)\right| \mathbf{1}_{\left\{\left\|F_{A, J}\right\|_{2, \mu} \leq R\right\}}\right) d \mu \leq A \sup _{\substack{\|h\|_{\infty, \mu} \leq 1 \\\|h\|_{2, \mu} \leq R / A}} \int_{X} \sup _{n \in I}\left|S_{n}(h)\right| d \mu .
$$

By substituting estimates (5.29) and (5.30) into (5.26), we can bound the first integral in the right-hand term of (5.25) as follows:

$$
\mathbf{E} \int_{B} \sup _{n \in I}\left|S_{n}\left(F_{A, J}\right)\right| d \mu \leq A S_{2}(I) e^{-R^{2} / 4 \alpha}(\sqrt{2}+\alpha-1)+A \sup _{\substack{\|h\| \infty, \mu \leq 1 \\\|h\|_{2, \mu} \leq R / A}} \int_{X} \sup _{n \in I}\left|S_{n}(h)\right| d \mu .
$$

Consider the second integral in the right-hand term of (5.25). We use Cauchy-Schwarz's inequality and the facts that $\mu\left(B^{c}\right) \leq \delta$ and

$$
\mathbf{E}\left\|F_{A, J}\right\|_{2, \mu} \leq \mathbf{E}\left\|F_{J}\right\|_{2, \mu} \leq 1
$$

to get the estimates

$$
\begin{aligned}
\mathbf{E} \int_{B^{c}} \sup _{n \in I}\left|S_{n}\left(F_{A, J}\right)\right| d \mu & \leq \sqrt{\mu\left(B^{c}\right)} \mathbf{E}\left\|\sup _{n \in I}\left|S_{n}\left(F_{A, J}\right)\right|\right\|_{2, \mu} \\
& \leq \sqrt{\delta} \sqrt{M} S_{1}(I) \mathbf{E}\left\|F_{A, J}\right\|_{2, \mu} \leq \sqrt{\alpha-1} e^{-A^{2} / 8 \alpha \sqrt{M}} S_{1}(I) .
\end{aligned}
$$

By inserting estimates (5.31) and (5.32) into (5.25), we next arrive at the estimate

$$
\begin{aligned}
\mathbf{E} \int \sup _{n \in I}\left|S_{n}\left(F_{A, J}\right)\right| d \mu \leq A S_{2}(I) e^{-R^{2} / 4 \alpha}(\sqrt{2}+\alpha-1) \\
\quad+A \sup _{\substack{\|h\|_{\infty, \mu \leq 1} \leq\left\|_{2, \mu} \leq R / A\\
\right\| h \|_{2, \mu}}} \sup _{n \in I}\left|S_{n}(h)\right| d \mu+\sqrt{\alpha-1} e^{-A^{2} / 8 \alpha \sqrt{M}} S_{1}(I) .
\end{aligned}
$$


Now we insert (5.24) and (5.33) into (5.14) and next use estimate (5.18). Picking $J$ arbitrarily in $\mathcal{J}^{*}$, we get the estimates

$$
\begin{aligned}
\gamma \mathbf{E} \sup _{n \in I} Z\left(S_{n}(f)\right) & \leq 6 \sqrt{\alpha M} S_{1}(I) \exp \left\{-A^{2} / 8\right\}+A S_{2}(I) e^{-R^{2} / 4 \alpha}(\sqrt{2}+\alpha-1) \\
& +A \sup _{\substack{\|h\|_{\infty, \mu \leq 1} \leq A \\
\|h\|_{2, \mu} \leq R / A}} \int_{X} \sup _{n \in I}\left|S_{n}(h)\right| d \mu+\sqrt{\alpha-1} e^{-A^{2} / 8 \alpha \sqrt{M} S_{1}(I) .}
\end{aligned}
$$

But $\alpha>1$ and $\gamma$ can be chosen arbitrarily close to 1 . Finally, we conclude that

$$
\begin{aligned}
\mathbf{E} \sup _{n \in I} Z\left(S_{n}(f)\right) \leq & 6 \sqrt{M} S_{1}(I) \exp \left\{-A^{2} / 8\right\}+A(\sqrt{2}) S_{2}(I) e^{-R^{2} / 4} \\
& +A \sup _{\substack{\|h\|_{\infty, \mu \leq 1} \\
\|h\|_{2, \mu \leq R / A}}} \int_{X} \sup _{n \in I}\left|S_{n}(h)\right| d \mu .
\end{aligned}
$$

Since this inequality is satisfied for any $f \in L^{\infty}(\mu)$ such that $\|f\|_{2, \mu}=1$, we easily deduce the claimed result by the continuity in the quadratic mean of $Z$.

\section{KaKUTANi-RokHLin'S LEMMA}

We conclude with this extremely useful tool in ergodic theory.

Lemma 6.1. If $T$ is aperiodic, then for every $\varepsilon>0$ and for every $n \geq 1$ there exists $F \in \mathcal{A}$ such that the sets $F, T^{-1}(F), \ldots, T^{-(n-1)}(F)$ are mutually disjoint and such that

$$
\mu\left(F \cup T^{-1}(F) \cup \cdots \cup T^{-(n-1)}(F)\right)>1-\varepsilon .
$$

Any set $F \in \mathcal{A}$ satisfying the conclusions of Lemma 6.1 is called an $(\varepsilon, n)$-Kakutani-Rokhlin set.

We illustrate its usefulness by establishing two divergence criteria for ergodic summation methods. The proof is based on an argument due to Deniel (see [7]). Let $\left\{w_{n, k}, 1 \leq k \leq\right.$ $n, n \geq 1\}$ be a triangular array of nonnegative reals and set $W_{n}=\sum_{k=1}^{n} w_{n, k}, n \geq 1$. Consider an automorphism $\tau$ from a probability space $(X, \mathcal{A}, \mu)$. Put for $f \in L^{0}(\mu)$,

$$
T_{n} f(x)=\frac{1}{W_{n}} \sum_{h=1}^{n} w_{n, h} f\left(\tau^{h} x\right) .
$$

Theorem 6.2. Let $\varphi: \mathbb{N} \rightarrow \mathbb{N}$ be such that $\lim _{n \rightarrow \infty} \varphi(n)=\infty$. Assume that there exists $\rho>0$ and an infinite sequence $\mathcal{N}$ of integers such that

$$
\min _{\varphi(n) \leq j \leq n-\varphi(n)}\left(\frac{1}{W_{n-j}} \sum_{k=n-j-\varphi(n)}^{n-j-1} w_{n-j, k}\right) \geq \rho
$$

for any $n \in \mathcal{N}$, and further that the series $\sum_{n \in \mathcal{N}} \varphi(n) / n$ converges. Let $0<\eta<\rho$. Then there exists $B \in \mathcal{A}$ with $0<\mu(B) \leq \eta$ such that $\limsup _{\mathcal{N} \ni n \rightarrow \infty} T_{n} \chi_{B} \geq \rho$ almost surely.

Remark 6.3. Assume that there exists a countable dense class $\mathcal{D}$ of functions from $L^{1}(\mu)$ such that $\left\{T_{n} f, n \in \mathcal{N}\right\}$ converges almost everywhere to $\int f d \mu$ for any $f \in \mathcal{D}$. Then if condition (6.1) is satisfied, there is no maximal inequality for the sequence $\left\{T_{n}, n \in \mathcal{N}\right\}$. Indeed, otherwise, by the Banach principle, we would have that $\left\{T_{n} f, n \in \mathcal{N}\right\}$ converges almost everywhere to $\int f d \mu$ for any $f \in L^{1}(\mu)$. Taking $f=\chi_{B}$, where $B$ is as in the proposition above, provides a contradiction. 
Now let $\left\{w_{k}, k \geq 1\right\}$ be a sequence of nonnegative reals and consider the ergodic sums

$$
A_{n} f(x)=\sum_{h=1}^{n} w_{h} f\left(\tau^{h} x\right)
$$

Theorem 6.4. Let $\varphi: \mathbb{N} \rightarrow \mathbb{N}$ be such that $\lim _{n \rightarrow \infty} \varphi(n)=\infty$. Assume that there exists $\rho>0$ and an infinite sequence $\mathcal{N}$ of integers such that

$$
\Delta_{n}:=\min _{1 \leq h \leq n-\varphi(n)}\left(\sum_{k=h}^{h+\varphi(n)} w_{k}\right) \rightarrow \infty
$$

for any $n \in \mathcal{N}$ as $n \rightarrow \infty$ along $\mathcal{N}$, and further that the series $\sum_{n \in \mathcal{N}} \varphi(n) / n$ converges. Let $0<\eta<\rho$. Then there exists $B \in \mathcal{A}$ with $0<\mu(B) \leq \eta$ such that $\limsup _{\mathcal{N} \ni n \rightarrow \infty} A_{n}\left(\chi_{B}\right)=\infty$ almost surely.

Proof of Theorem 6.2. There is no loss of generality in assuming that

$$
\sum_{n \in \mathcal{N}} \varphi(n) / n \leq \eta
$$

By Rokhlin's lemma, for any $\varepsilon>0$ and any integer $N$, there exists $A \in \mathcal{A}$ such that $A, T A, \ldots, T^{N-1} A$, are pairwise disjoint and $1-\varepsilon \leq N \mu(A) \leq 1$. By applying it for $N=n$ and $\varepsilon=\varphi(n) / n$, we conclude that for each $n \in \mathcal{N}$ there exists $A_{n} \in \mathcal{A}$ such that $A_{n}, \tau A_{n}, \ldots, \tau^{n-1} A_{n}$ are mutually disjoint and $\mu\left(\sum_{u=0}^{n-1} \tau^{u} A_{n}\right)=n \mu\left(A_{n}\right) \geq 1-\varphi(n) / n$. Let

$$
B_{n}=\sum_{n-\varphi(n) \leq u<n} \tau^{u} A_{n} \quad \text { and } \quad D_{n}=\sum_{\varphi(n) \leq j<n-\varphi(n)} \tau^{j} A_{n} .
$$

Then

and

$$
\mu\left(B_{n}\right) \leq \varphi(n) \mu\left(A_{n}\right) \leq \frac{\varphi(n)}{n}
$$

$$
\mu\left(D_{n}\right) \geq \frac{n-2 \varphi(n)}{n}\left(1-\frac{\varphi(n)}{n}\right) \geq\left(1-2 \frac{\varphi(n)}{n}\right)^{2} \geq 1-4 \frac{\varphi(n)}{n} .
$$

Now let $0 \leq \ell<n-\varphi(n)$. Since $\tau^{\ell} x \in B_{n}$ iff $x \in \tau^{u-\ell} A_{n}$ for some $n-\varphi(n) \leq u<n$, we can write

$$
\chi_{B_{n}}\left(\tau^{\ell} x\right)=\sum_{n-\varphi(n) \leq u<n} \chi_{\left\{\tau^{u-\ell} A_{n}\right\}}(x)=\sum_{n-\varphi(n)-\ell \leq v<n-\ell} \chi_{\left\{\tau^{v} A_{n}\right\}}(x) .
$$

Let $\ell=n-\varphi(n)-\lambda$ with $1 \leq \lambda<n-\varphi(n)$. Then

$$
\chi_{B_{n}}\left(\tau^{n-\varphi(n)-\lambda} x\right)=\sum_{\lambda \leq v<\lambda+\varphi(n)} \chi_{\left\{\tau^{v} A_{n}\right\}}(x) .
$$

Since $\varphi(n) / n \rightarrow 0$ when $n \rightarrow \infty$ along $\mathcal{N}$, we have the inequality $2 \varphi(n) \leq n$ once $n$ is large. Fix some $\varphi(n) \leq j<n-\varphi(n)$ and pick $x \in \tau^{j} A_{n}$. If we choose $\lambda$ so that $\lambda \leq j<\lambda+\varphi(n)$, by letting $v=j$ in the equation above, we see that $\tau^{n-\varphi(n)-\lambda} x \in B_{n}$.

Thus, $x \in \tau^{j} A_{n}$ and $\lambda \in\{j-\varphi(n)+1, j-\varphi(n)+2, \ldots, j\}$ imply that

$$
\tau^{n-\varphi(n)-\lambda} x \in B_{n} .
$$


Consequently, if $x \in \tau^{j} A_{n}$, then

$$
\begin{aligned}
T_{n-j} \chi_{B_{n}}(x) & =\sum_{k=1}^{n-j} w_{n-j, n-j-k} \chi_{B_{n}}\left(\tau^{n-j-k} x\right) \\
& \geq \sum_{k=1}^{\varphi(n)} w_{n-j, n-j-k} \chi_{B_{n}}\left(\tau^{n-j-k} x\right) \\
& =\sum_{\lambda=j-\varphi(n)+1}^{j} w_{n-j, n-\varphi(n)-\lambda} \chi_{B_{n}}\left(\tau^{n-\varphi(n)-\lambda} x\right) \\
& =\sum_{\lambda=j-\varphi(n)+1}^{j} w_{n-j, n-\varphi(n)-\lambda}=\sum_{k=1}^{\varphi(n)} w_{n-j, n-j-k} .
\end{aligned}
$$

By the assumption made,

$$
\frac{1}{W_{n-j}} \sum_{k=1}^{\varphi(n)} w_{n-j, n-j-k} \geq \min _{\varphi(n) \leq j \leq n-\varphi(n)}\left(\frac{1}{W_{n-j}} \sum_{k=1}^{\varphi(n)} w_{n-j, n-j-k}\right) \geq \rho .
$$

Note that $n-j>\varphi(n)$. Thus, on $D_{n}$,

$$
\sup _{m>\varphi(n)} T_{m}\left(\chi_{B_{n}}\right) \geq \rho .
$$

Set

$$
E=\bigcup_{n \in \mathcal{N}} B_{n} \quad \text { and } \quad F_{N}=\bigcap_{\substack{n \in \mathcal{N} \\ n \geq N}} D_{n}
$$

We observe that $\mu\left(F_{N}\right) \geq 1-4 \sum_{\substack{n \in \mathcal{N} \\ n \geq N}} \varphi(n) / n \rightarrow 1$ as $N \rightarrow \infty$. Thus, on $F_{N}$,

$$
\limsup _{\mathcal{N} \ni n \rightarrow \infty} T_{n}\left(\chi_{E}\right) \geq \rho .
$$

Further, $\mu(E) \leq \sum_{n \in \mathcal{N}} \varphi(n) / n<\eta$. This establishes Theorem 6.2.

Proof of Theorem 6.4. We start with (6.3) which here becomes

$$
A_{n-j} \chi_{B_{n}}(x) \geq \sum_{k=1}^{\varphi(n)} w_{n-j-k}
$$

and next modify the previous proof as follows:

$$
\sum_{k=1}^{\varphi(n)} w_{n-j-k} \geq \min _{\varphi(n) \leq j \leq n-\varphi(n)}\left(\sum_{k=1}^{\varphi(n)} w_{n-j-k}\right) \geq \min _{1 \leq h \leq n-\varphi(n)}\left(\sum_{k=h}^{h+\varphi(n)} w_{k}\right)=\Delta_{n} .
$$

Thus, $\sup _{m>\varphi(n)} A_{m}\left(\chi_{B_{n}}\right) \geq \Delta_{n}$ on $D_{n}$. Therefore, on $F_{N}$,

$$
\limsup _{\mathcal{N} \ni n \rightarrow \infty} T_{n} \chi_{E}=\infty \text {. }
$$

Further, $\mu(E) \leq \sum_{n \in \mathcal{N}} \varphi(n) / n<\eta$. 
Acknowlegments: The author thanks the referee for valuable suggestions and Christophe Cuny for useful comments.

\section{REFERENCES}

1. J. Bourgain, "Problems of almost everywhere convergence related to harmonic analysis and number theory," Israel J. Math., 71, 97-127. (1990)

2. J. Bourgain, "Almost sure convergence and bounded entropy," Israel J. Math., 63, 79-95 (1988).

3. J. Bourgain, "An approach to pointwise ergodic theorems," in: Geometric Aspects of Functional Analysis (1986/87), Lecture Notes Math., 1317 (1988), pp. 204-223.

4. A. Bellow and R. Jones, "A Banach principle for $L^{\infty}, "$ Adv. Math., 120, 155-172 (1996).

5. D. L. Burkholder, "Maximal inequalities as necessary conditions for almost everywhere convergence," Z. Wahrsch. Verw. Geb., 3, 75-88 (1964).

6. I. Berkes and M. Weber, "On series $\sum c_{k} f(k x)$ and Khinchin's conjecture," Israel J. Math., 201, 593-609 (2014).

7. Y. Deniel, "On the a.s. Cesàro- $\alpha$ convergence for stationary of orthogonal sequences," $J$. Theor. Probab., 2, 475-485 (1989).

8. R. M. Dudley, "Sample functions of the Gaussian process," Ann. Probab., 1, 66-103 (1973).

9. R. M. Dudley, "The size of compact subsets of Hilbert space and continuity of Gaussian processes," J. Funct. Anal., 1, 290-330 (1967).

10. A. Garsia, Topics in Almost Everywhere Convergence, Markham Publ. Co., Chicago (1970).

11. A. Ya. Helemskiı̌ and G. M. Henkin, "Embeddings of compacta into ellipsoids," Vestnik Moskov. Univ., Ser. I, Matem. Mekh., No. 2, 3-12 (1963).

12. A. N. Kolmogorov, "Asymptotic characteristics of some completely bounded metric spaces," Dokl. Akad. Nauk SSSR, 108, 585-589 (1956).

13. A. N. Kolmogorov, "Sur les fonctions harmoniques conjuguées et les séries de Fourier," Fund. Math., 7, 23-28 (1925).

14. A. N. Kolmogorov, "Une série de Fourier-Lebesgue divergente presque partout," Fund. Math., 4, 324-328 (1923).

15. A. N. Kolmogorov, "Une série de Fourier-Lebesgue divergente presque partout," $C . R$. Acad. Sci. Paris, Sér. I, Math., 183, 1327-1329 (1926).

16. A. N. Kolmogorov and V. M. Tikhomirov, " $\varepsilon$-entropy and $\varepsilon$-capacity of sets in function spaces," Usp. Mat. Nauk, 14, 1-86 (1959).

17. M. T. Lacey, "Carleson's theorem, proof, complements, variations," Publ. Math., 48, 251307 (2004).

18. M. T. Lacey, "The return time theorem fails on infinite measure preserving systems," Ann. Inst. Henri Poincaré, 33, 491-495 (1997).

19. J. Lamperti, "On the isometries of certain function-spaces," Pacific J. Math., 8, 459-466 (1958).

20. E. Lesigne, "On the sequence of integer parts of a good sequence for the ergodic theorem," Comment. Math. Univ. Carolin., 36, 737-743 (1995).

21. M. Lifshits and M. Weber, "Oscillations of the Gaussian Stein's elements," in: High Dimensional Probability (Oberwolfach, 1996), Progr. Probab., 43, (1998), pp. 249-261.

22. M. Lifshits and M. Weber, "Tightness of stochastic families arising from randomization procedure," in: Asymptotic methods in probability and statistics with applications (St. Petersburg, 1998), Stat. Ind. Technol., Birkhäuser, Boston (2001), pp. 143-158.

23. G. G. Lorentz, "Metric entropy and approximation," Bull. Amer. Math. Soc., 72, 903-937 (1966). 
24. M. Marcus and G. Pisier, "Characterizations of almost surely $p$-stable random Fourier series and strongly continuous stationary processes," Acta Math., 152, 245-301 (1984).

25. R. A. Raimi, "Compact transformations and the $k$-topology in Hilbert space," Proc. Amer. Math. Soc., 6, 643-646 (1955).

26. J. M. Rosenblatt and M. Wierdl, "Pointwise ergodic theorems via harmonic analysis," in: Proc. Conf. Ergodic Theory and its Connections with Harmonic Analysis, Alexandria, Egypt, Cambridge Univ. Press (1994), pp. 3-151.

27. S. Sawyer, "Maximal inequalities of weak type," Ann. Math., 84, 157-174 (1996).

28. D. Schneider and M. Weber, "Une remarque sur un théorème de Bourgain," in: Séminaire de Probabilités, XXVII, Lect. Notes Math., 1557 (1993), pp. 202-206.

29. E. M. Stein, "On limits of sequences of operators," Ann. Math., 74, 140-170 (1961).

30. M. Talagrand, Upper and Lower Bounds for Stochastic Processes, Erg. der Math. und ihrer Grenzgeb., 60, Springer, Berlin-Heidelberg (2014).

31. M. Talagrand, "Applying a theorem of Fernique," Ann. Inst. H. Poincaré, 32, 779-799 (1996).

32. M. Weber, Dynamical Systems and Processes, Eur. Math. Soc. Pub. House, IRMA Lect. in Math. and Theor. Physics, 14 (2009).

33. M. Weber, Entropie Métrique et Convergence Presque Partout, Travaux en Cours, 58, Hermann, Paris (1998).

34. M. Weber, "Entropy numbers in $L^{p}$-spaces for averages of rotations," J. Math. Kyoto Univ., 37, 689-700 (1997).

35. M. Weber, "The Stein randomization procedure," Rendiconti Math., Ser. VII, 16, 569-605 (1996).

36. M. Weber, "Coupling of the GB set property for ergodic averages," J. Theor. Probab., 9, 105-112 (1996).

37. M. Weber, "GB and GC sets in ergodic theory," in: Probability in Banach Spaces, 9 (Sandjberg, 1993), Progr. Probab., 35, Birkhäuser Boston, Boston MA (1994), pp. 129151.

38. M. Weber, "Opérateurs réguliers sur les espaces $L^{p}$," in: Séminaire de probabilités XXVII, Lect. Notes Math., 1557 (1993) pp. 207-215. 